\title{
Analysis and Application of an Underwater Optical-Ranging System
}

by

John Gerald Kusters, Jr.

B.S., California State Polytechnic University, Pomona (1984)

Submitted in partial fulfillment of the

requirements for the degree of

OCEAN ENGINEER

and

MASTER OF SCIENCE IN OCEAN ENGINEERING

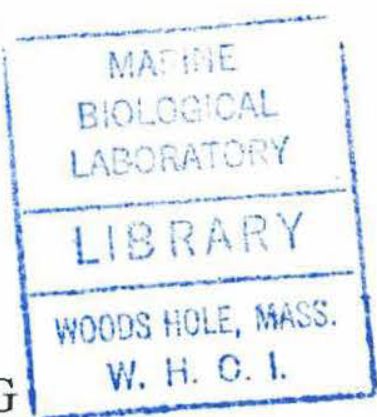

at the

MASSACHUSETTS INSTITUTE OF TECHNOLOGY

and the

WOODS HOLE OCEANOGRAPHIC INSTITUTION

September 1992

(C) John Gerald Kusters, Jr. 1992

The author hereby grants to MIT and WHOI permission to reproduce and distribute copies of this thesis document in whole or part.

Signature of Author

Joint Program in Oceanographic Engineering
Massachusetts Institute of Technology/
Woods Hole Oceanographic Institution

August 28, 1992

Certified by

W. Kenneth Stewart, Jr.

Woods Hole Oceanographic Institution

Thesis Supervisor

Accepted by

Arthur B. Baggeroer

Chairman, Joint Committee for Applied Ocean Science and Engineering

Massachusetts Institute of Technology/

Woods Hole Oceanographic Institution

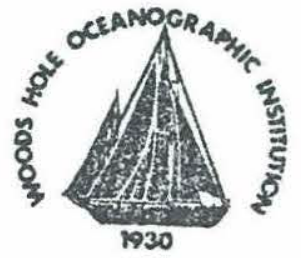




\title{
Analysis and Application of an Underwater Optical-Ranging System
}

\author{
by \\ John Gerald Kusters, Jr. \\ Submitted to the Massachusetts Institute of Technology / \\ Woods Hole Oceanographic Institution \\ Joint Program in Applied Ocean Science and Engineering \\ on August 28, 1992 in partial fulfillment \\ of the requirements for the degree of \\ Ocean Engineer and \\ Master of Science in Ocean Engineering
}

\begin{abstract}
In order to provide a high-resolution underwater-ranging capability for scientific measurement, a commercially available optical-ranging system is analyzed for performance and feasibility. The system employs a structured-lighting technique using a laser-light plane and single-camera imaging system. The mechanics of determining range with such a system are presented along with predicted range error. Controlled testing of the system is performed and range error is empirically determined. The system is employed in a deep-sea application, and its performance is evaluated. The measurements obtained are used for a scientific application to determine seafloor roughness for very-high-spatial frequencies (greater than 10 cycles/meter). Use and application recommendations for the system are presented.
\end{abstract}

Thesis Supervisor: W. Kenneth Stewart, Jr.

Woods Hole Oceanographic Institution 


\section{Acknowledgements}

I would like to make special mention of those without whose assistance this thesis would not have been possible and give my gratitude to the following:

- My thesis advisor, Ken Stewart, whose unbelievable patience and faith went above and beyond any previous records set.

- The Deep Submergence Lab's Engineering Team, whose skill and at-sea expertise made a major portion of this work possible. I would like to especially thank Andy Bowen and Bob Elder.

- The Crest '91 ROV Pilots, Will Sellers, Dave Lavolvo, and Skip Gleason, whose incredible skill and adept abilities made the close-range laser surveys possible. Their special consideration during Crest '91 is greatly appreciated.

- The Crest '91 Chief Scientists, John Delaney and Bob Ballard, for the priceless bottom time needed.

- My officemates, Tad Snow and Hanumant Singh, for always offering a listening ear no matter how bad the idea may have been and their valuable advice.

- The Sysjan, Marty Marra, for my innumerable interruptions and his forbearance in always answering the question, 'How do I?'

- The local expert, Jon Howland, for his time, experience, and dedicated interest.

- The U.S. Navy and Oceanographer of the Navy for their sponsorship and this incredible opportunity to work in this field and perform uninhibited research.

Lastly, a special thanks to my parents, John and Marie, who always said the right things and gave me the encouragement to succeed and, above all else, to my wife, HollyAnn, for continuing in that tradition and providing me with her ever loving support. 


\section{Contents}

$\begin{array}{ll}\text { ABSTRACT } & 2\end{array}$

$\begin{array}{ll}\text { Chapter } 1 \text { INTRODUCTION } & 11\end{array}$

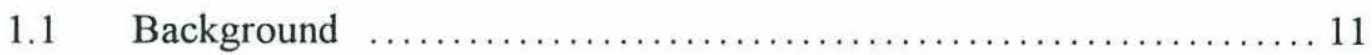

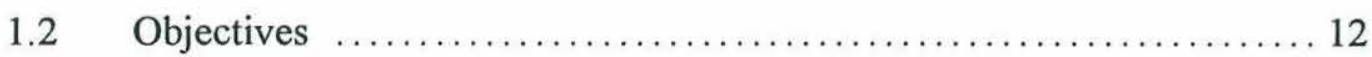

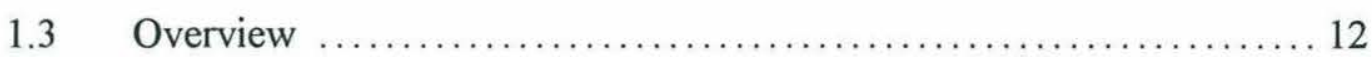

Chapter 2 HIGH-RESOLUTION UNDERWATER RANGING 14

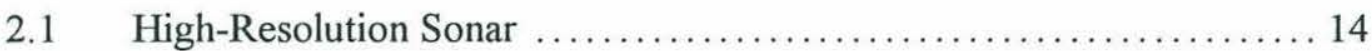

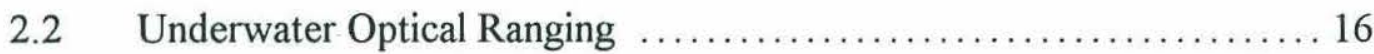

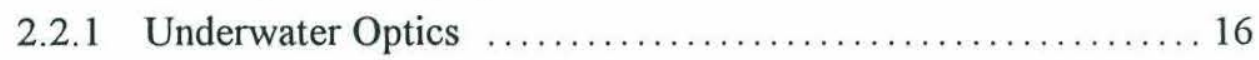

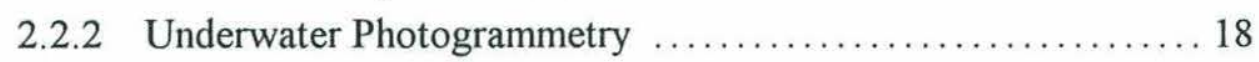

2.2.3 Laser-Ranging Systems $\ldots \ldots \ldots \ldots \ldots \ldots \ldots \ldots \ldots \ldots \ldots \ldots \ldots \ldots \ldots \ldots$

2.2.3.1 Laser-Radar Systems (LIDAR) ............... 19

2.2.3.2 Amplitude-Modulated Ranging Systems ............ 20

2.2.3.3 Structured-Lighting Techniques ................ 22

Chapter 3 LASER-PLANE STRUCTURED LIGHTING 23

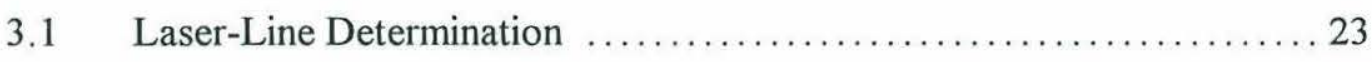

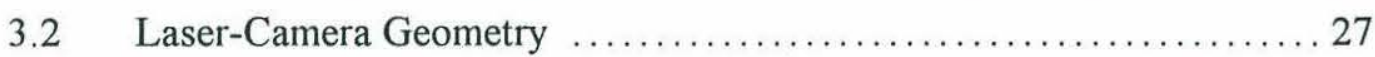

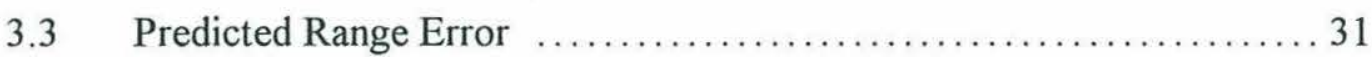

3.3.1 Lens-Aberration Error $\ldots \ldots \ldots \ldots \ldots \ldots \ldots \ldots \ldots \ldots \ldots \ldots \ldots \ldots \ldots \ldots \ldots \ldots$

3.3.2 Laser-Line Localization Error $\ldots \ldots \ldots \ldots \ldots \ldots \ldots \ldots \ldots \ldots \ldots \ldots \ldots \ldots \ldots \ldots$

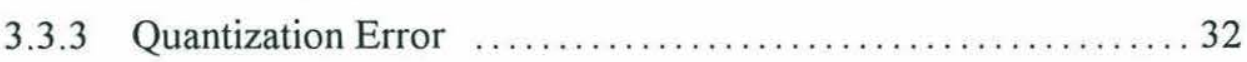

3.3.4 Total Predicted Range Error ........................ 33

3.4 The SPOTSCAN 2D Optical-Ranging System ................ 36

Chapter 4 CONTROLLED TESTING 38

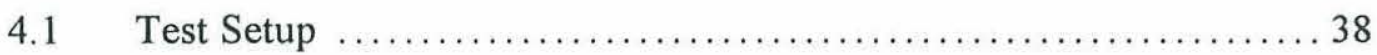




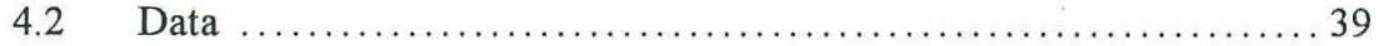

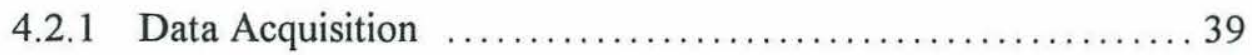

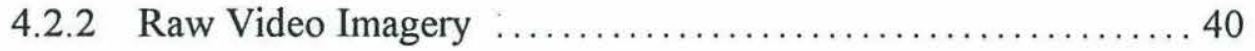

4.2.3 Processed Profiles ................................ 41

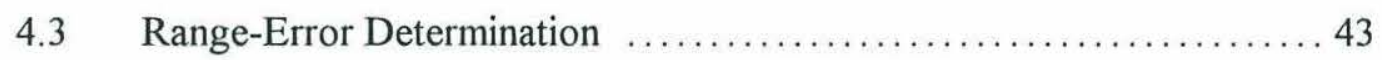

4.3.1 Tank and Target Model $\ldots \ldots \ldots \ldots \ldots \ldots \ldots \ldots \ldots \ldots \ldots \ldots \ldots$

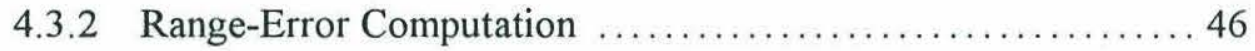

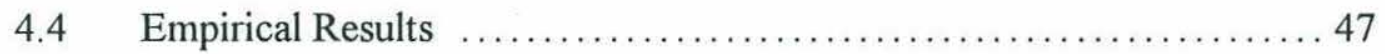

4.5 Comparison of Empirical and Analytical Error .................. 49

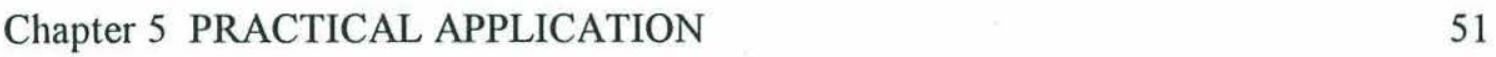

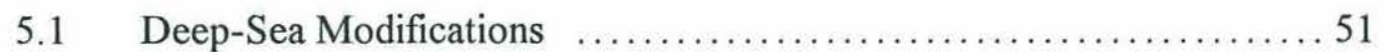

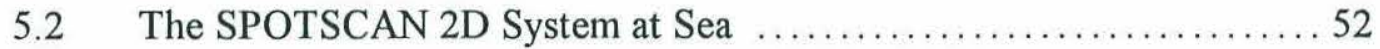

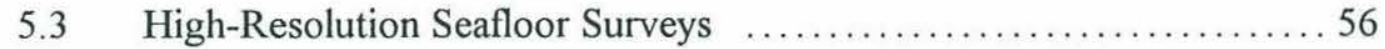

5.4 High-Spatial-Frequency Seafloor-Roughness Measurements ........ 58

5.5 Power Spectral Density from Laser Profiles $\ldots \ldots \ldots \ldots \ldots \ldots \ldots \ldots$

5.5.1 Profile Selection and Resampling $\ldots \ldots \ldots \ldots \ldots \ldots \ldots \ldots 60$

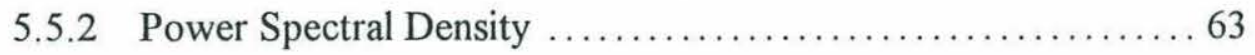

$\begin{array}{ll}\text { Chapter } 6 \text { CONCLUSIONS } & 67\end{array}$

6.1 The Practicality of Underwater Optical Ranging $\ldots \ldots \ldots \ldots \ldots \ldots \ldots 67$

6.2 Recommendations and Improvements $\ldots \ldots \ldots \ldots \ldots \ldots \ldots \ldots \ldots \ldots$ 


\section{List of Figures}

Figure 2.1 Spectral attenuation of optical and acoustical radiation $\ldots \ldots \ldots \ldots \ldots \ldots$

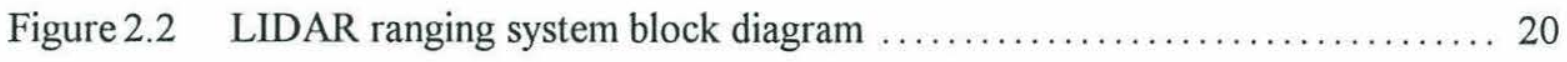

Figure 2.3 Beam-modulated ranging system block diagram $\ldots \ldots \ldots \ldots \ldots \ldots \ldots \ldots 21$

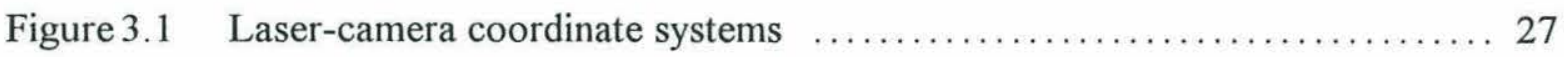

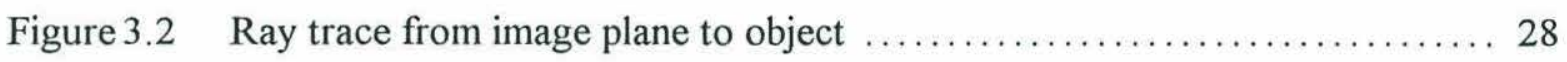

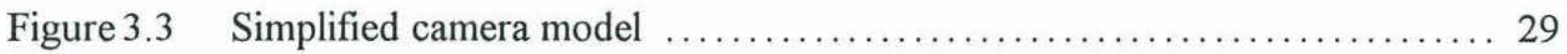

Figure 3.4 Object localization within the laser plane $\ldots \ldots \ldots \ldots \ldots \ldots \ldots \ldots \ldots \ldots \ldots \ldots \ldots$

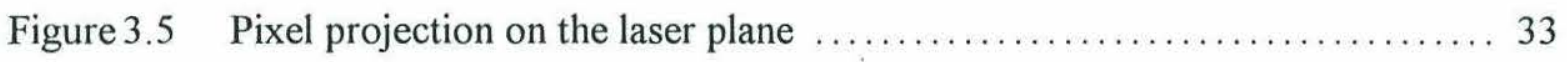

Figure 3.6 Predicted range error for SPOTSCAN 2D $\ldots \ldots \ldots \ldots \ldots \ldots \ldots \ldots \ldots \ldots \ldots \ldots \ldots \ldots \ldots \ldots \ldots$

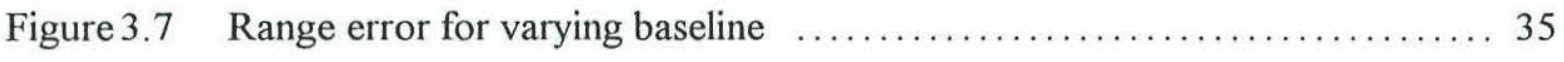

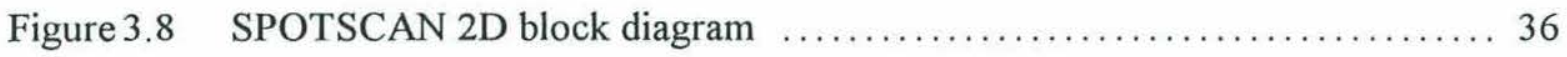

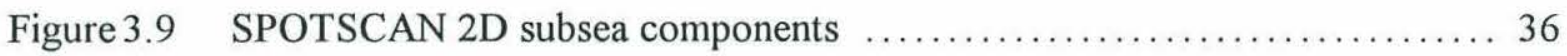

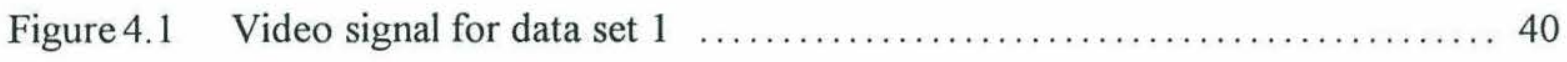

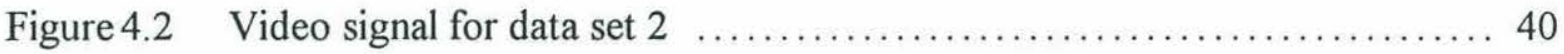

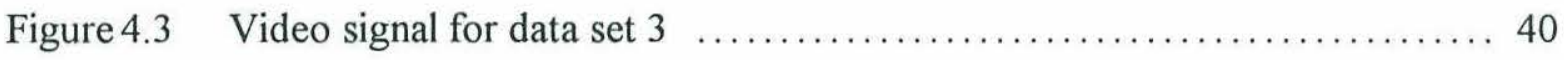

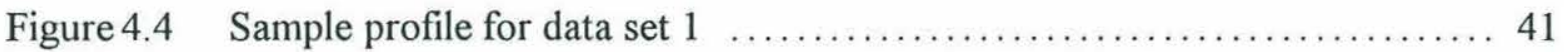

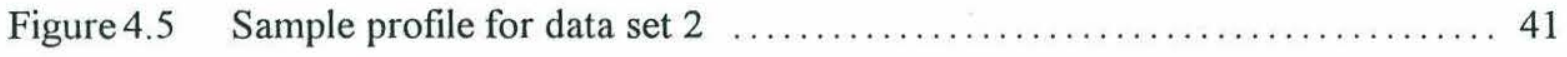

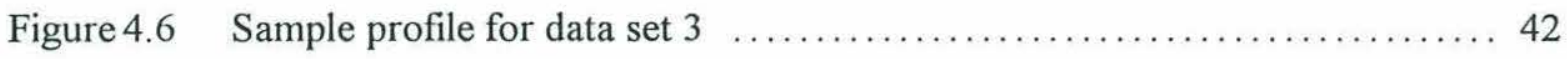

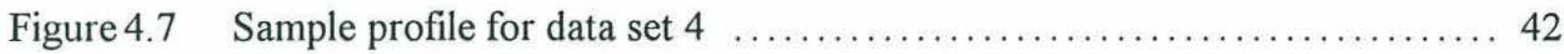

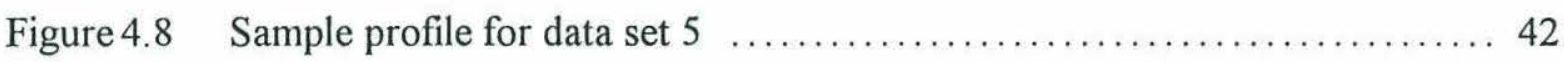

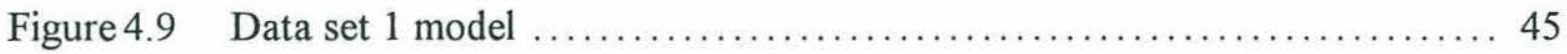

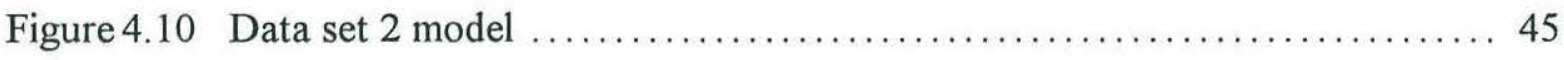

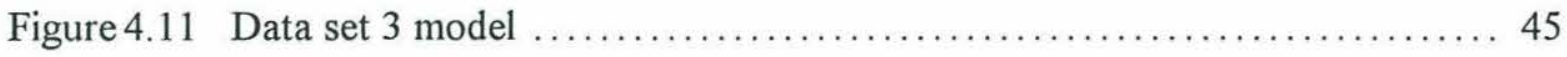

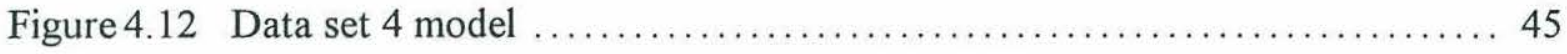

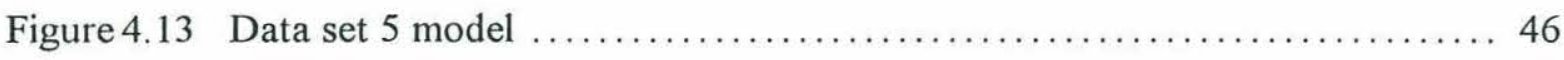

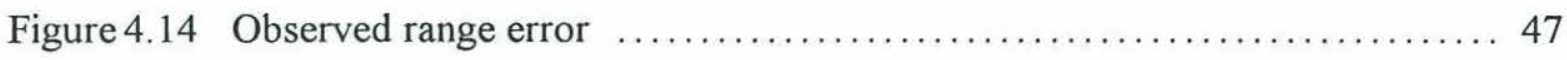

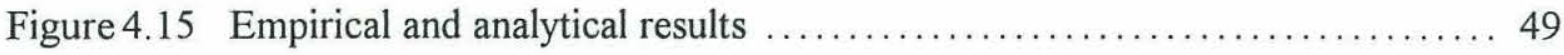




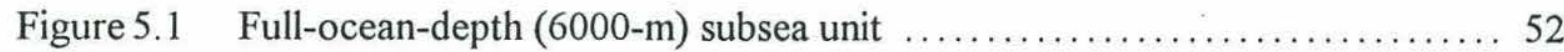

Figure 5.2 Remotely operated vehicle - Jason $\ldots \ldots \ldots \ldots \ldots \ldots \ldots \ldots \ldots \ldots \ldots \ldots \ldots \ldots \ldots \ldots \ldots$

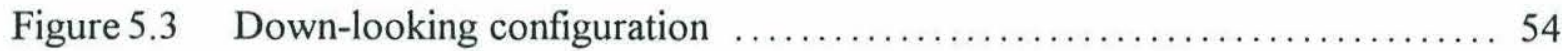

Figure 5.4 Detail of down-looking configuration $\ldots \ldots \ldots \ldots \ldots \ldots \ldots \ldots \ldots \ldots \ldots$

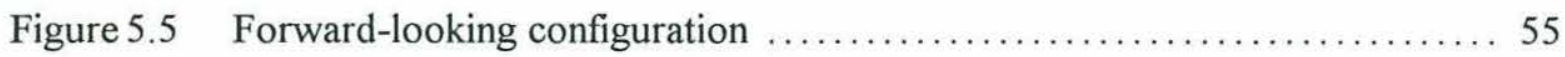

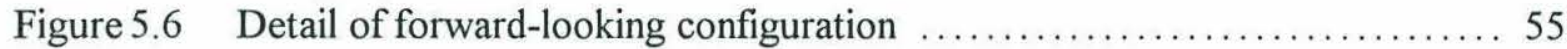

Figure 5.7 Typical bottom profile obtained during Crest ' $91 \ldots \ldots \ldots \ldots \ldots \ldots \ldots$

Figure 5.8 Typical forward profile obtained during Crest ' $91 \ldots \ldots \ldots \ldots \ldots \ldots \ldots$

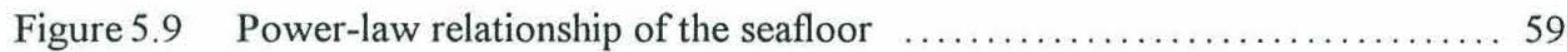

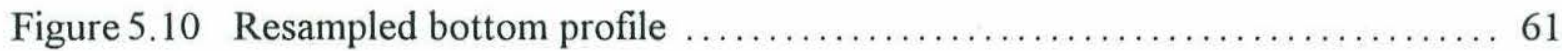

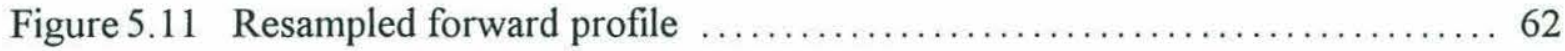

Figure 5.12 Resampled close-range forward profile $\ldots \ldots \ldots \ldots \ldots \ldots \ldots \ldots \ldots \ldots \ldots \ldots \ldots \ldots \ldots$

Figure 5.13 Power spectrum of bottom profiles $\ldots \ldots \ldots \ldots \ldots \ldots \ldots \ldots \ldots \ldots \ldots \ldots \ldots \ldots \ldots \ldots$

Figure 5.14 Power spectrum of forward profiles $\ldots \ldots \ldots \ldots \ldots \ldots \ldots \ldots \ldots \ldots \ldots \ldots \ldots \ldots \ldots \ldots \ldots$

Figure 5.15 Composite power spectrum of laser profiles $\ldots \ldots \ldots \ldots \ldots \ldots \ldots \ldots \ldots$

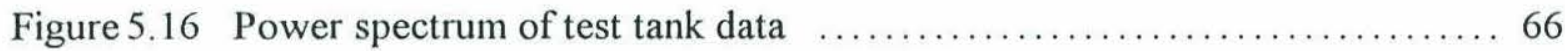




\section{List of Tables}

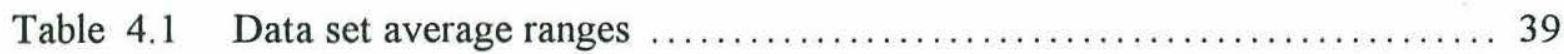

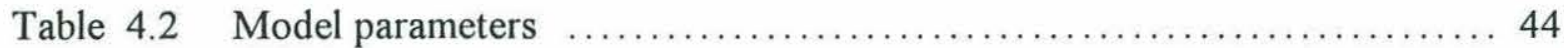




\section{Nomenclature}

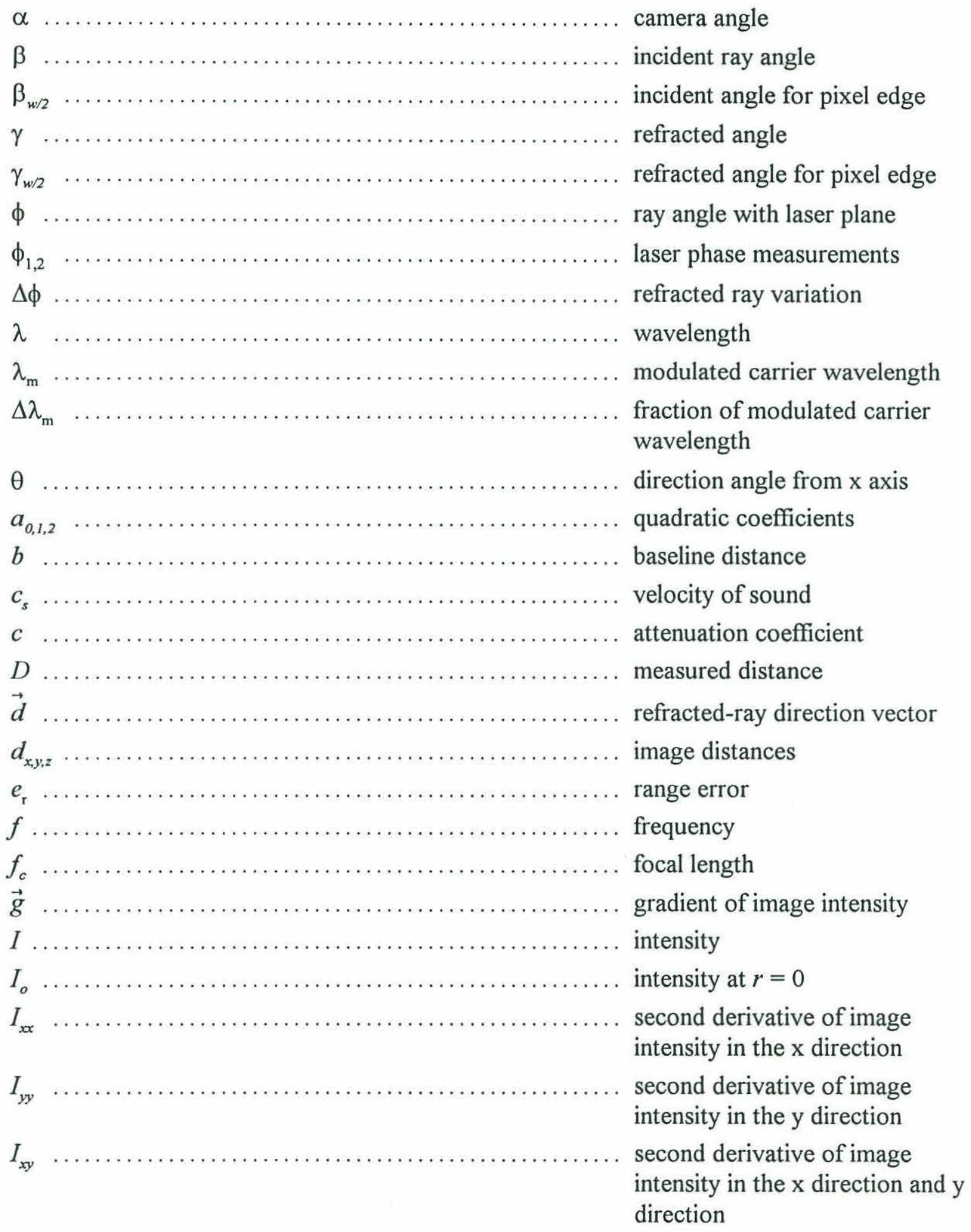




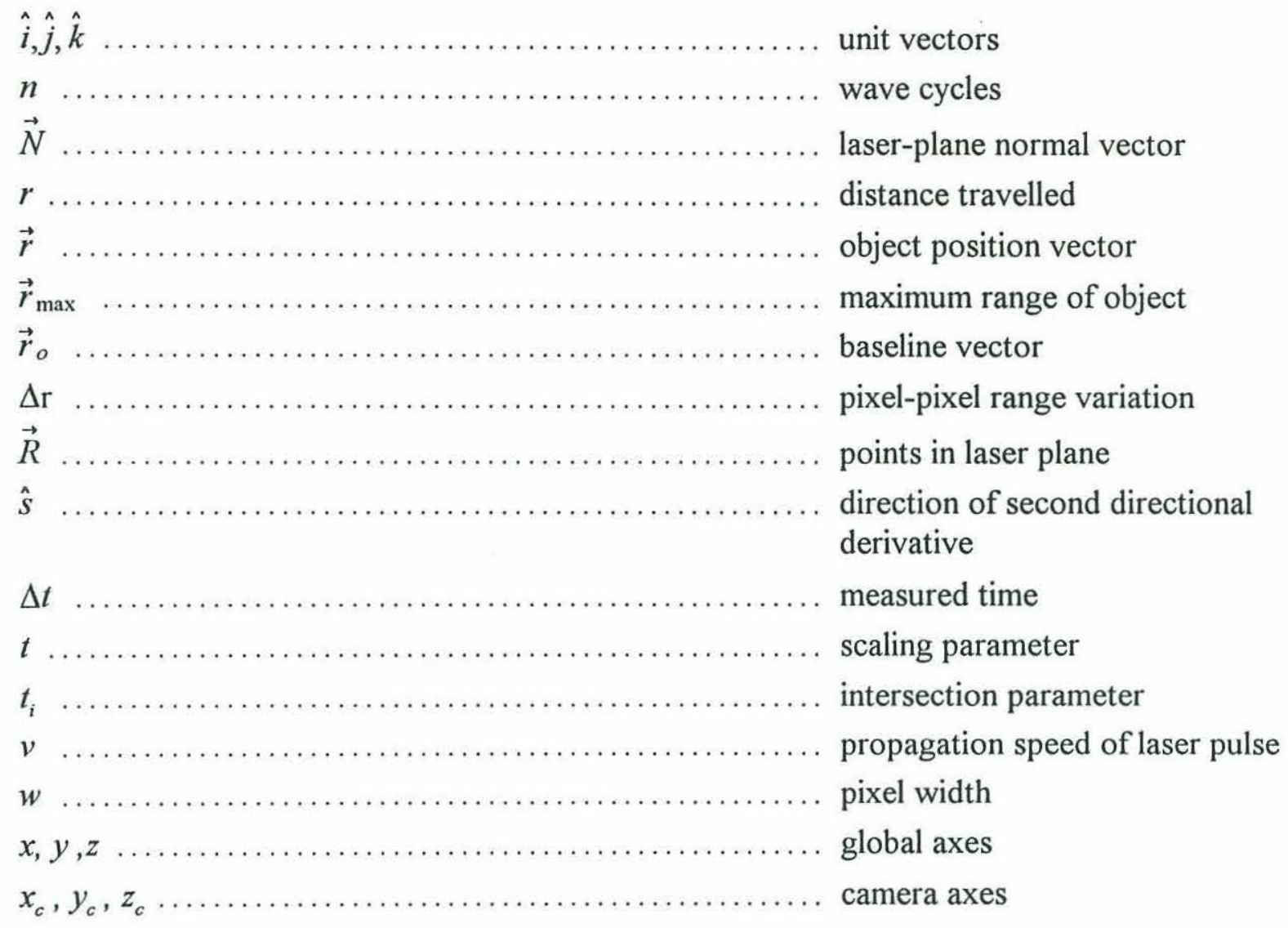




\section{Chapter 1}

\section{INTRODUCTION}

\subsection{Background}

Remote sensing of the underwater environment takes on many forms and has many applications. Typical ocean surveys can include measurements of varied parameters from physical properties of the seawater itself to inferences about the biologics present. A fundamental measurement of ocean surveying, which in itself has many forms, is distance. Underwater ranging has typically been performed acoustically and, for most cases, this is the preferred method. However, as higher resolutions are desired, alternative optical methods provide advantages which favor this option.

The varied disciplines studying the ocean today place a high demand on the engineer to develop and produce reliable and effective techniques for measuring their particular parameters. The acoustician who develops ranging instrumentation requires detailed information about material roughness to determine acoustic-backscatter properties. From the geologist who asks for an ability to measure the seafloor at scales from kilometers to meters and smaller to the archaeologist who wants detailed information on submerged artifacts, accurate and dependable ranging measurements, covering a wide spectrum of resolutions, are in high demand. To provide for one end of these requirements, optical-ranging methods capable of delivering high resolutions in the millimeter range have been developed. Although several of these methods have been proposed and implemented, few are available on a wide-commercial scale, and thus, fewer still have been analyzed and studied for feasibility in scientific applications. To help fulfill the requirement of a very-high-resolution underwater range-measurement technique this thesis offers a detailed analysis of a commercially available optical-ranging method. 


\subsection{Objectives}

The aim of this thesis is to determine if a commercially available very-high-resolution ranging system can be used for precise scientific range measurements underwater. One such system, the SPOTSCAN 2D from Seatex A/S of Trondheim Norway, was available for testing and analysis. The system employs a structured-lighting technique capable of millimeter resolution at very close range $(<2 \mathrm{~m})$. A detailed description is presented in Chapter 3 .

The overall thesis objectives can be broken down into distinct tasks as follows:

- Determine range-measurement performance of the system.

- Test the system in a controlled environment and empirically determine performance.

- Employ the system in an at-sea scientific application and evaluate its usefulness.

The broad goal is to fully understand system performance and functionality so the system can be used to its full capabilities. In addition, scientific conclusions drawn from measurements of the environment must take into account careful consideration of the instrumentation used. With this in mind, this thesis analyzes a particular instrument and reports on its performance and capabilities. The system is studied in both a controlled-testing environment and in an at-sea application. Alternative systems capable of similar performance are briefly presented for comparison and to widen the scope of selection. It is hoped that the work performed here can assist others in choosing a system for high-resolution measurements in a deep-sea environment.

\subsection{Overview}

In the following chapter a brief overview is given of available underwater high-resolution ranging methods. A comparison is made of acoustical versus optical methods for very-high-resolution ranging and conclusions drawn about the advantages of optical systems. The issues associated with underwater optics and photogrammetry are discussed, and possible solutions are presented for the problems encountered. Available underwater optical-ranging techniques are then reviewed with the advantages and disadvantages of each method described. 
Chapter 3 presents the details of structured-lighting techniques using a laser-light plane and single-camera system. The basic geometry is discussed and a simple model of the system is developed. A general approach is taken to develop equations for determining object range using photogrammetric techniques. These equations are then used to predict range error based on quantization levels in the digital-camera system and errors in locating the laser-light plane. The model is then applied to the SPOTSCAN 2D laser system and results are presented. Engineering and functional details of this specific laser system are also presented.

In Chapter 4 controlled testing of the laser system is presented with the observed range-error performance. The test setup and conditions are described including detail of the targets used. A review of the data gathered is presented and then the range-error determination method is discussed. Examples of the test models are shown. The empirical results are then presented along with the previously determined analytical range error.

Chapter 5 includes the at-sea performance of a modified SPOTSCAN 2D. The system was employed at depths in excess of $2200 \mathrm{~m}$ during Crest '91, a research cruise conducted near the Juan de Fuca Ridge in the Northeastern Pacific Ocean. The required system modifications for deep-sea capability are discussed along with the configurations used during these seafloor surveys. A brief discussion of a seafloor-roughness stochastic model is presented and the approximate power-law relationship of the seafloor power spectral density function is demonstrated using several types of data obtained during Crest '91. The technicalities and problems with using the laser system for these types of measurements are discussed and a workable solution is presented. The seafloor power spectral density function for very-high-spatial frequencies is computed from the laser profiles. Conclusions about system performance and practicality of at-sea use are made in Chapter 6. 


\section{Chapter 2}

\section{HIGH-RESOLUTION UNDERWATER RANGING}

High-resolution remote-range measurements of the underwater environment have typically been made with high-frequency sonar. This yields resolutions in the centimeter range, but further increases require impractical frequencies. To attain these higher resolutions, optical techniques provide a viable alternative. In this chapter the major issues associated with optical techniques for underwater measurements are presented along with details of various optical-ranging methods in use today.

\subsection{High-Resolution Sonar}

Range measurements using acoustic techniques are most commonly performed by time-of-flight measurements. Attainable resolutions depend on the wavelength and width of the insonifying pulse [1]. At higher resolutions, shorter pulses and correspondingly shorter wavelengths are required. Frequency $f$ is directly determined by the speed of sound $c_{s}$ and the wavelength $\lambda$ from

$$
f=\frac{c_{s}}{\lambda} .
$$

Therefore, shorter wavelengths require higher frequencies. However, as frequency is increased attenuation also increases monotonically [2]. Consequently, higher resolutions have higher attenuation and a corresponding limit on attainable range. In comparison, optical attenuation dictates a narrow transmission window around wavelengths of $500 \mathrm{~nm}$ (blue-green part of the spectrum). Figure 2.1 shows the relationship of attenuation and wavelength for both optical and acoustic radiation $[3,4,5,6]$. Both source and target radiation are exponentially attenuated. A characteristic parameter is the beam-attenuation coefficient, $c$ $(1 / \mathrm{m})$, where the beam intensity, $I$, is described by the Lambert-Beer law,

$$
I=I_{o} e^{-c r}
$$


where $r$ is the distance traveled, and $I_{\mathrm{o}}$ is the intensity at $r=0$.

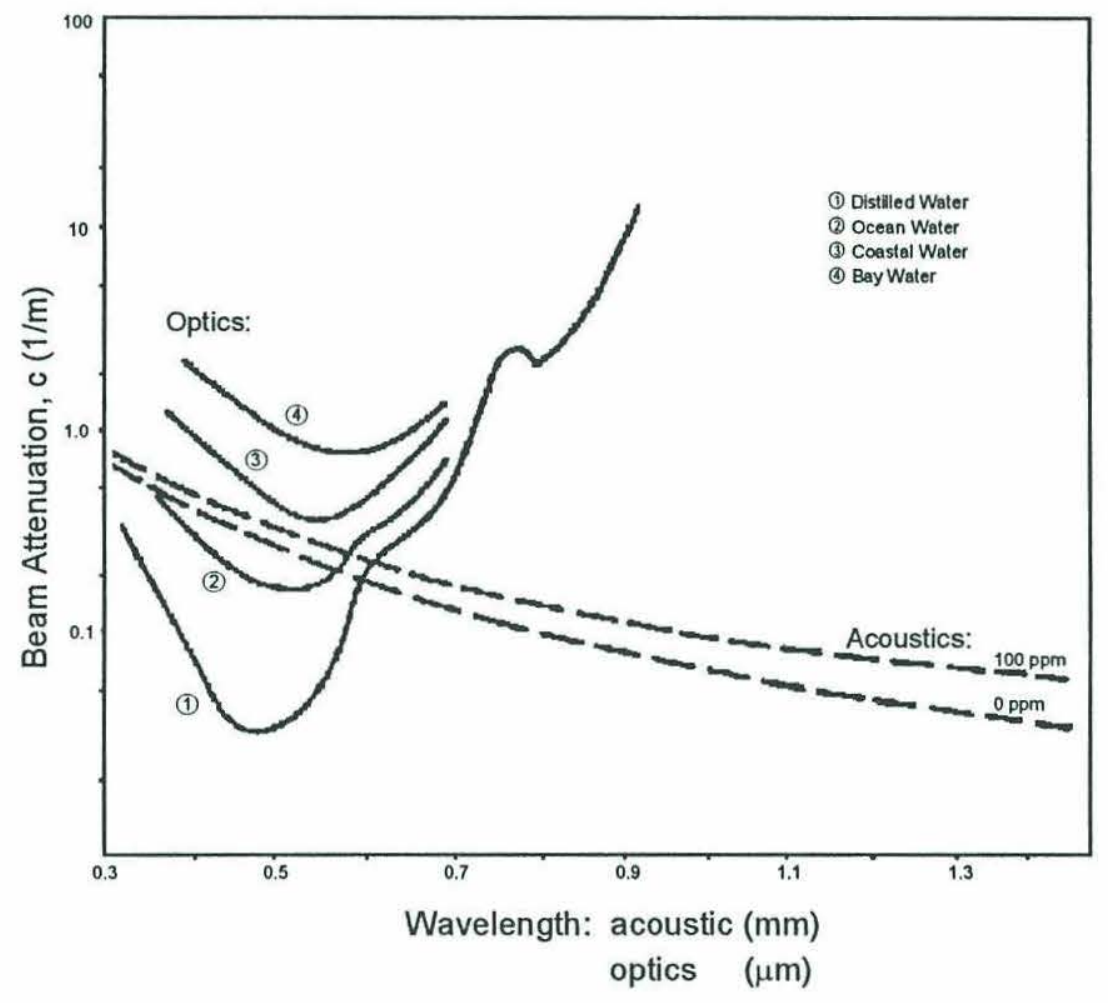

Figure 2.1: Spectral attenuation of optical and acoustical radiation [6] 


\subsection{Underwater Optical Ranging}

There are two basic methods for obtaining range information with light: time-of-flight and triangulation. Triangulation methods are possible using standard imaging techniques (stereoscopy) and do not necessarily require the use of lasers, however, the matching process required can be difficult [7]. Stereoscopic systems determine range measurements indirectly through the use of displaced dual-images using exact position information of either a single-camera or a dual-camera system. The object must be located in each image then ray traces are calculated using photogrammetric techniques. The range is determined by triangulation of the ray traces. As previously stated, these methods in themselves are complicated and require extensive processing techniques to solve the correspondence problem of locating the same object in two separate images. The matching process can be performed manually but this presents obvious disadvantages. In addition, the method does not provide direct range measurement.

This thesis concentrates on single-image ranging techniques, which require precise lighting conditions to determine range information. The extremely accurate directional capabilities and distinct wavelengths of lasers make them ideal light sources for triangulation methods in underwater applications. Of the optical ranging methods available, this technique has currently proved to be the most viable for underwater applications [5].

Time-of-flight and phase-comparitor systems have been established as highly effective techniques in atmospheric conditions. Time-of-flight systems use a pulsed-laser and a time-elapsed device to measure the distance a laser-light pulse travels, then infer range from the measured time. Phase-comparitor systems use an amplitude-modulated laser beam and measure the difference between received and transmitted phases to determine the range. These systems are presented here for comparison but, in general, they have shortcomings when applied to the underwater environment.

\subsubsection{Underwater Optics}

Electromagnetic radiation has long been an effective tool for sensing techniques and in the transfer of information. However, for the most part these uses have been developed for 
in-air applications, since high attenuation of electromagnetic radiation in water has deterred its development as a tool for this environment. Only in the past decade or two have attempts been made to utilize this source for underwater applications. Communication techniques have begun to show some promise [8] and, more recently, remote-ranging methods have demonstrated commercial success [9]. Underwater-imaging systems employing scanning lasers for illumination have demonstrated dramatic results in increasing visible ranges $[10,11,12]$ and, although not direct-range-measurement systems, the images produced can be used through photogrammetric (stereoscopic) techniques to provide useful distance information.

Optical sensing in an underwater environment involves some of the same principles as acoustic sensing. A target is illuminated by a radiative source and the backscattered energy is detected and processed to determine the parameter of interest. Attenuation due to absorbtion and scattering [3] of the radiated energy as it travels through the water greatly affects the transmission range both to the target and back to the receiver. In addition, the dependence of refraction on wavelength causes dispersion, which produces an effect similar to chromatic aberration of lenses and thereby reduces resolution [13]. This problem can be overcome through the use of a single wavelength of coherent light, making laser applications attractive.

Scattering by suspended material in the water increases attenuation and, in the case of imaging systems, a loss of resolution and contrast [14]. As a rule, in ocean waters away from coastal regions particle concentrations decrease with increasing depth and for deep-water applications ( $>300 \mathrm{~m})$ their effect is minimal [3]. However, remote surveying of the seafloor, particularly with optical methods, requires low altitudes that can lead to disturbances of the sediment and a correspondingly significant scattering problem. Methods exist for enhancing poor quality images resulting from these effects [15] but, in general, they are best avoided because of difficult implementation and interpretation of results. 


\subsubsection{Underwater Photogrammetry}

As defined by the American Society of Photogrammetry [16]:

Photogrammetry is the art, science, and technology of obtaining reliable information about physical objects and the environment through processes of recording, measuring, and interpreting photographic images and patterns of electromagnetic radiant energy and other phenomena.

In this sense, underwater photogrammetry is the application of these methods to the situation where radiative source, receiver, and target are present in the underwater environment. Photogrammetric methods for obtaining range measurements involve a wide spectrum of mathematical principles and methods [17]. A detailed presentation of these principles is left to the references and instead only the notable anomalies encountered in the underwater environment are discussed.

The principal difference in using a camera-lens system in the underwater environment is the presence of media with different refractive indices on either side of the lens. The principal and nodal points no longer coincide when the object space has a different refractive index than the image space [14]. This situation produces severe geometric distortions that can be overcome through the use of a special in-water lens or a front-port system between the water and in-air lens. The former presents further difficulties if the system must be used in varying underwater conditions since the refractive index for water can vary significantly with salinity, pressure, and temperature $[4,14]$. Front-port systems form a virtual image that behaves as the object for the camera. Rays from the true object are refracted at both the water-port interface and again at the port-air interface. The effect is a significant reduction in the field of view, however, the advantages and simplicity of the design make it a likely choice. Domed ports and fully corrective ports can provide distinct advantages over a simple planar port but their complexity and cost can be prohibitive.

\subsubsection{Laser-Ranging Systems}

The use of lasers in optical-ranging systems makes time-of-flight optical methods workable and can greatly simplify the design and implementation of triangulation systems. In the underwater environment the use of a single wavelength of coherent light avoids dispersion 
problems encountered from the dependency of refraction on frequency. Advances in solid-state-laser design have made the use of these systems possible in underwater applications [18]. Due to the high absorption by ocean water of electromagnetic radiation in all but the blue-green part of the visible spectrum, it is desirable to use wavelengths around $500 \mathrm{~nm}$. In addition, for underwater applications concerns about size, power requirements, data-transmission limitations, and reliability place demands on lasers systems that have only recently been met [5]. Neodymium:Yttrium-Aluminum-Garnet (Nd:YAG) diode-pumped lasers using frequency doubling [19] have been invented and meet the requirements for an underwater laser-ranging system. The commercial system studied in this thesis uses such a laser operating in a continuous mode.

\subsubsection{Laser-Radar Ranging Systems (LIDAR)}

Laser radar or lidar systems typically employ a pulsed-laser transmitter and photodetector receiver $[19,20,21]$. Figure 2.2 [20] shows the basic components in a typical laser timed-pulse distance-measurement system. The transmitted pulse is detected and opens an electronic gate that connects an oscillator to a high-speed counter. The gate is subsequently closed when the return signal is detected by the receiving optics. The range is obtained by measuring the time required for the light to travel from the transmitter to the target and back. The distance is given by

$$
D=\frac{v t}{2}
$$

where $D$ is the distance measurement, $t$ is the measured time, and $v$ the speed of propagation of the laser pulse, corrected for ambient conditions. Available underwater technology limits pulsed-laser ranges to approximately $100 \mathrm{~m}$ due to attenuation [10]. At these ranges the transit time is extremely small. Errors in this method are mainly related to accurate measurement of this short transit time. In addition, difficulties presented by the underwater environment from refraction and scattering make this method hard to implement for high-resolution underwater ranging. 


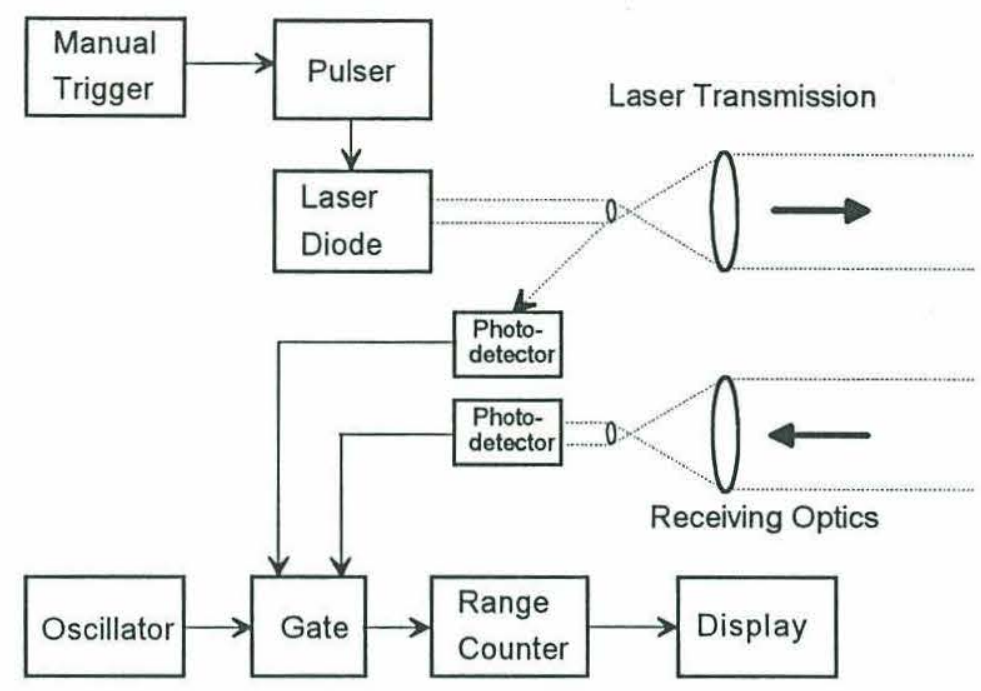

Figure 2.2: LIDAR ranging system block diagram [20]

\subsubsection{Amplitude-Modulated Ranging Systems}

In this method the beam amplitude is modulated and the phase shift of the reflected light is measured. The device transmits a laser beam modulated with a sinusoidal wave form. When the beam strikes an object, a portion of the light is reflected back to the receiver. The phase of the returned signal is measured and compared with the reference phase. The relative difference is proportional to the distance traveled and can be expressed in terms of the modulation wavelength $\lambda_{m}$ as

$$
2 D=n \lambda_{m}+\Delta \lambda_{m}
$$

where $D$ is the distance traveled, $n$ is the integer number of completed cycles, and $\Delta \lambda_{m}$ is the fraction of a wavelength left over. By measuring the phase of the transmitted wave, $\phi_{1}$, and that of the returned signal, $\phi_{2}, \Delta \lambda_{m}$ can be determined from

$$
\Delta \lambda_{m}=\frac{\phi_{2}-\phi_{1}}{360^{\circ}} \lambda_{m}
$$

The distance $D$ is made up of two components and in a phase-comparitor system only $\Delta \lambda_{m}$ can be determined. To find the total distance the $n \lambda_{m}$ term must be resolved. This is referred to as resolving the ambiguity of the phase comparison and is commonly performed by increasing the 
wavelength in multiples of 10 so a coarse measurement of the distance can be made and subsequently converged to the required resolution.

Figure 2.3 [20] shows the essential components for a phase-comparison beam-modulated ranging system. The laser beam and carrier signal are mixed together in a modulator and the resultant beam is transmitted. The reflected beam re-enters the instrument and demodulation takes place. Comparison of the reference and transmitted signals produces the required phase difference, which is used along with the resolved ambiguity to determine the range.

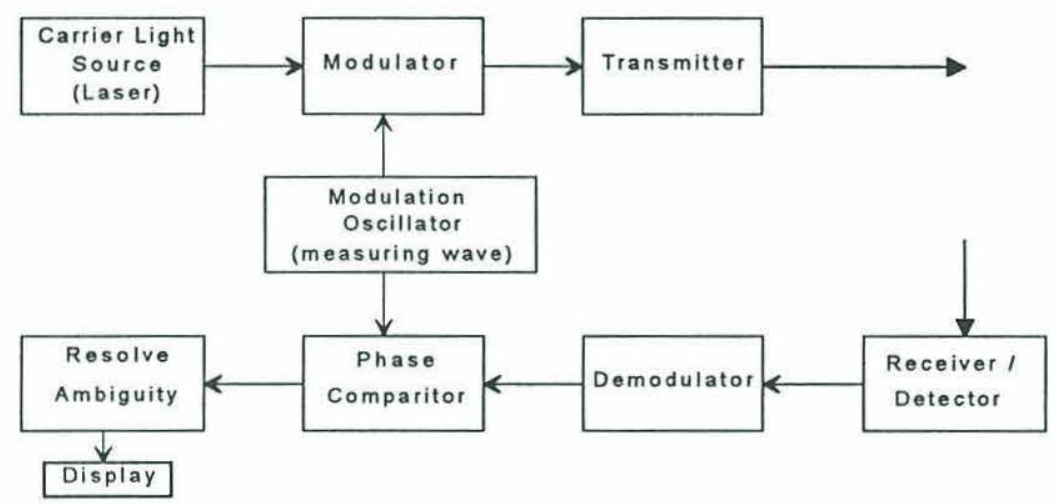

Figure 2.3: Beam-modulated ranging system block diagram [20] 


\subsubsection{Structured-Lighting Techniques}

Structured-lighting techniques offer an attractive alternative to time-of-flight methods because there is a relatively simple implementation that allows processing of a line instead of a single point. This approach is based on the light-strip sensing technique $[22,23,24]$ and incorporates photogrammetric algorithms [25]. The process projects a plane of light onto an object and acquires the resultant line's image. The position and geometry of the light source are known and, through ray tracing, points on the line are determined by intersection with the light plane. Since the light plane has finite thickness the assumption of a plane can pose difficulties. However, by employing line-finding algorithms [7] a thin line corresponding to the plane can be determined. Beginning with a thin plane improves system performance, suggesting the use of a laser. Another source of error is generated by quantization of the line's image, posing a limitation on the resolving capability of the system. A detailed discussion and analysis is presented in the following chapter. 


\section{Chapter 3}

\section{LASER-PLANE STRUCTURED LIGHTING}

In this chapter the details of laser-plane structured lighting are presented. The image-processing techniques for laser-line determination are discussed, and a second-directional-derivative line-finding routine is derived. The basic geometric principles for determining range with structured lighting are presented, and a simple model of a structured-lighting system employing a laser-light plane is developed. System range error is analytically predicted using the model. A fully functional laser-plane structured-lighting ranging system, the SPOTSCAN 2D from Seatex A/S, is then described.

\subsection{Laser-Line Determination}

In a laser-plane structured-lighting system the intersection of the laser plane and an object in its path produces a line in the camera image. By determining the exact position of a line in the image, the corresponding points in the laser plane can be determined through photogrammetric techniques. The laser-line image will typically contain noise generated by ambient illumination. A line-finding routine should reject those pixels corresponding to noise and retain the pixels illuminated by the laser light. This suggest thresholding techniques since the laser light amplitude is expected to be significantly greater than other sources. However, since the laser-line image is typically wider than a single pixel, thresholding in itself will not yield the desired results because it produces a line wider than a single pixel and voids the thin-plane assumption required by the geometry for object localization.

The light-stripe method [22] thresholds the pixel intensities then uses their center of mass as the line determination. This method is viable and will produce correct results, however it only considers intensity values in a one-dimensional sense. The image is broken into discrete horizontal or vertical lines and thresholding is applied to the pixels within this 
line. The resultant pixels are then used in the center of mass determination to locate the laser line. Another method that uses a two-dimensional search for the line will tend to produce better results since it considers more of the surrounding pixels in line determination than the light-stripe method.

A method that uses two-dimensional information of the image for line localization is the second-directional-derivative line-finding routine [7]. A bright line can be thought of as a crest in the intensity values of an image. The second directional derivative is most negative along the direction orthogonal to the crest. In this direction the first derivative of intensity values changes sign and the second derivative is less than zero. The first directional derivative is zero at the top of the crest, however, it is unlikely to locate this exact point.

It is more feasible instead to search for zero-crossings (where the sign changes from positive to negative or vice versa). The directional derivative $\frac{\partial}{\partial s}$ in the direction specified by the angle $\theta$ with the $x$ axis is given by [26]

$$
\frac{\partial}{\partial s}=\cos \theta \frac{\partial}{\partial x}+\sin \theta \frac{\partial}{\partial y}
$$

Applying this twice to the intensity $I$ gives the second directional derivative

$$
\frac{\partial^{2} I}{\partial s^{2}}=\frac{1}{2}\left(I_{x x}+I_{y y}\right)+\frac{1}{2}\left(I_{x x}-I_{y y}\right) \cos 2 \theta+I_{x y} \sin 2 \theta
$$

The direction in which this is maximized or minimized is found by differentiating with respect to $\theta$ and setting to zero

$$
\begin{gathered}
\frac{\partial}{\partial \theta}\left(\frac{\partial^{2} I}{\partial s^{2}}\right)=0 \\
\text { or } \\
-\left(I_{x x}-I_{y y}\right) \sin 2 \theta+2 I_{x y} \cos 2 \theta=0 \\
\text { or } \\
\tan 2 \theta=\frac{2 I_{x y}}{I_{x x}-I_{y y}}
\end{gathered}
$$

resulting in

$$
\sin 2 \theta=\frac{ \pm 2 I_{x y}}{\sqrt{\left(I_{x x}-I_{y y}\right)^{2}+4 I_{x y}^{2}}}
$$




$$
\cos 2 \theta=\frac{ \pm\left(I_{x x}-I_{y y}\right)}{\sqrt{\left(I_{x x}-I_{y y}\right)^{2}+4 I_{x y}^{2}}}
$$

Substituting into (3.2) gives

$$
\begin{aligned}
& \left.\frac{\partial^{2} I}{\partial s^{2}}\right|_{\text {max,min }}=\frac{1}{2}\left(I_{x x}+I_{y y}\right) \pm \frac{\left[\left(I_{x x}-I_{y y}\right)^{2}+4 I_{x y}^{2}\right]}{2 \sqrt{\left(I_{x x}-I_{y y}\right)^{2}+4 I_{x y}^{2}}} \\
& \text { or } \\
& \left.\frac{\partial^{2} I}{\partial s^{2}}\right|_{\max , \min }=\frac{1}{2}\left[\left(I_{x x}+I_{y y}\right) \pm \sqrt{\left(I_{x x}-I_{y y}\right)^{2}+4 I_{x y}^{2}}\right]
\end{aligned}
$$

Since the radical term is positive, the direction of the most negative second directional derivative is given by the negative root in (3.9) or

$$
\cos 2 \theta=\frac{-\left(I_{x x}-I_{y y}\right)}{\sqrt{\left(I_{x x}-I_{y y}\right)^{2}+4 I_{x y}^{2}}}
$$

So

$$
\begin{aligned}
& |\cos \theta|=\frac{\sqrt{S+\left(I_{y y}-I_{x x}\right)}}{\sqrt{2 S}} \\
& \text { and } \\
& |\sin \theta|=\frac{\sqrt{S+\left(I_{x x}-I_{y y}\right)}}{\sqrt{2 S}}
\end{aligned}
$$

where

$$
S=\sqrt{\left(I_{x x}-I_{y y}\right)^{2}+4 I_{x y}^{2}}
$$


Since traversing the line orthogonally, regardless of direction $(\theta$ or $\theta+\pi)$, yields the most negative second directional derivative, $\theta$ is defined so that $\cos \theta>0$. Therefore, the sign of $\sin \theta$ is given by $-\operatorname{sign}\left(\mathrm{I}_{\mathrm{xy}}\right)$ since $S$ is positive and

$$
\sin 2 \theta=2 \sin \theta \cos \theta=\frac{-2 I_{x y}}{S}
$$

The direction is then given by the unit vector $\hat{s}$ where

$$
\hat{s}=\frac{1}{\sqrt{2 S}}\left\{\sqrt{S+\left(I_{y y}-I_{x x}\right)} \hat{i}-\operatorname{sign}\left(I_{x y}\right) \sqrt{S+\left(I_{x x}-I_{y y}\right)} \hat{j}\right\}
$$

The first directional derivative of the intensity can be considered as the dot product of the intensity gradient $\vec{g}$ and the direction vector $\hat{s}$, since

$$
\hat{s}=\cos \theta \hat{i}+\sin \theta \hat{j}
$$

and

$$
\frac{\partial I}{\partial s}=\cos \theta \frac{\partial I}{\partial x}+\sin \theta \frac{\partial I}{\partial y}
$$

So for

$$
\begin{aligned}
& \vec{g}=I_{x} \hat{i}+I_{y} \hat{j} \\
& \frac{\partial I}{\partial s}=\hat{s} \cdot \vec{g}
\end{aligned}
$$

A search for the zero-crossings of the dot product of the intensity gradient and the direction of the most negative second directional derivative locates lines within the image [7].

Filtering of the image in either of the cases presented can reduce noise prior to line detection yielding better results. Filtering blurs the line, however, the methods presented can counter this effect, since they are adapted to determine thin lines from thicker smeared or blurred lines. If ambient levels produce noise above or near the amplitude of laser illumination then a two-dimensional routine such as the one presented here should be employed to reduce false localization of points not associated with the laser line.

A significant drawback of the two-dimensional line-finding routine or prior filtering of the image is the increased computational resources required. The center-of-mass thresholding technique is generally much faster and produces satisfactory results for most applications. 


\subsection{Laser-Camera Geometry}

Once the laser-line position has been determined within the image, basic geometric principles can be used to determine its location within the laser plane. Since the line represents the intersection of the laser plane and the object to be ranged, its location corresponds to object range. To determine range using laser-plane structured lighting, the coordinate system shown in Figure 3.1 is established. The global coordinate axes are placed such that the laser plane lies in the $x-z$ plane as shown. The camera coordinate axes are aligned with the origin at the cameras center of projection, $x_{c}$ axis along the optical axis, and the $z_{c}$ axis parallel to the laser's (global) $z$ axis. The camera is rotated about its $z_{c}$ axis through an angle, $\alpha$, so the field of view is optimal for the range-finding system.

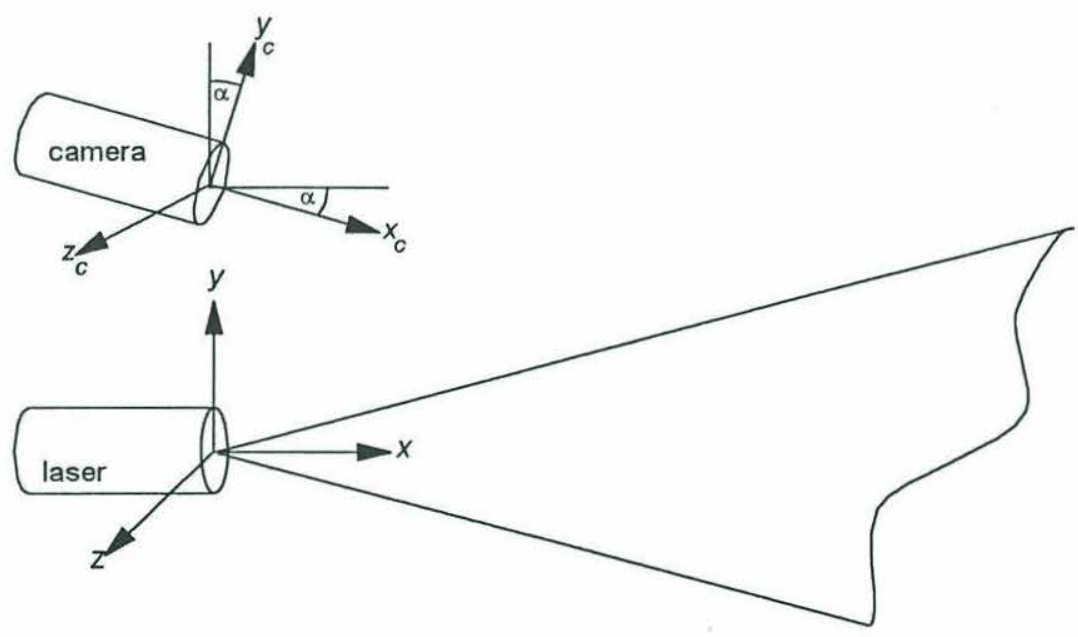

Figure 3.1: Laser-camera coordinate systems 


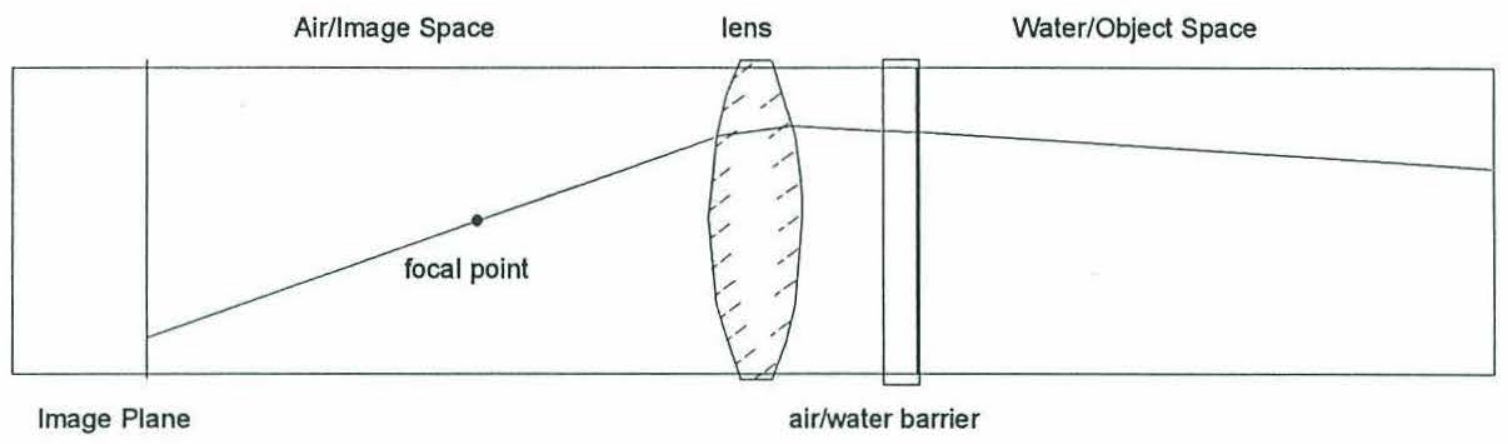

Figure 3.2: Ray trace from image plane to object

An object lying in the laser plane reflects its light and the corresponding image can be used to determine the object's location within the laser plane. This is accomplished by tracing the ray of light from its point of illumination in the image plane through the camera/air space, the camera lenses, and the water medium to the intersection with the laser plane [4] (see Figure 3.2). Each point in the image plane defines a ray through the camera-lens focal point. After accounting for refraction at the various interfaces, this ray defines a line in the global space of Figure 3.1. The determination of this ray's position in the global coordinate system is crucial in locating the corresponding object in the laser plane. Errors in the ray position are caused by inaccuracy in estimating the various interactions of its path.

The image of an object point produced by a lens system will not be a distinct point but a blur, even in the plane of best focus [27]. These image imperfections are called aberrations and are measured quantitatively by the size of the blur. In the inverse problem considered for structured-lighting techniques, due to lens aberrations, the image point must be considered a small disc when projected on the laser plane. To simplify the photogrammetry involved a pinhole-camera model is used with a single air-water interface (i.e., lens thickness is negligible), and the lens aberrations are considered as errors in determining the ray's position within the global coordinate-system. The lens-aberration effects are further discussed in the following section on error analysis . Figure 3.3 demonstrates the model. 


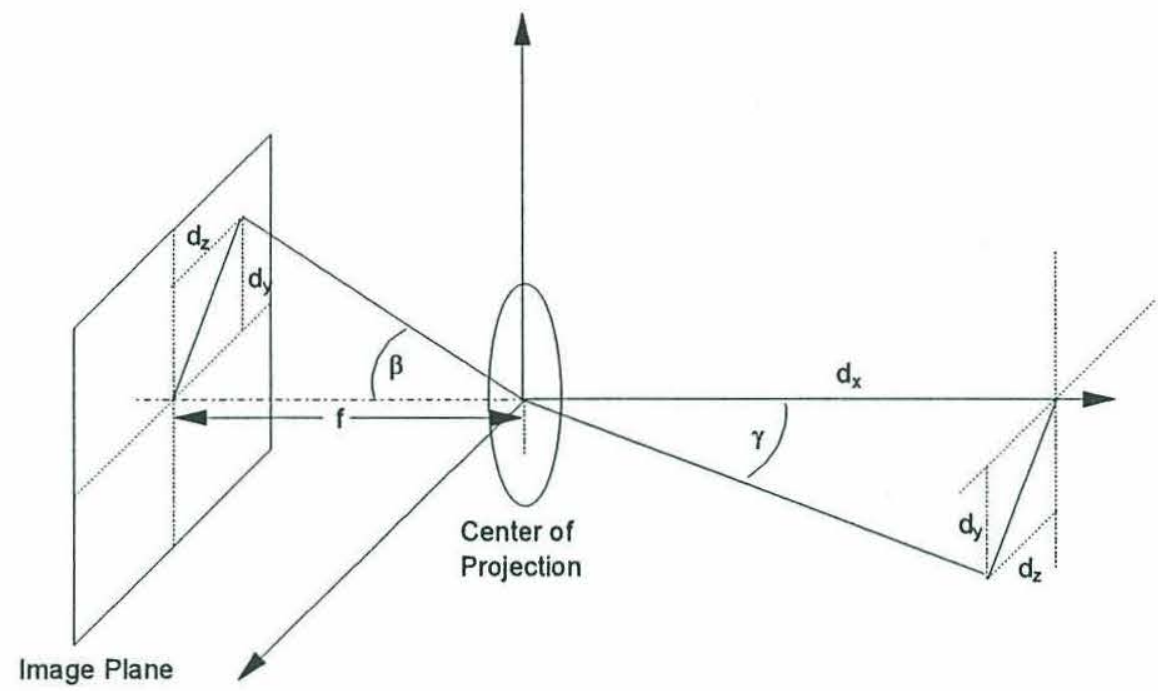

Figure 3.3: Simplified camera model

A point in the image plane is defined by the location $\left(d_{y}, d_{z}\right)$. The angle $\beta$ is the incident angle on the interface and is given by

$$
\beta=\arctan \left(\frac{\sqrt{d_{y}^{2}+d_{z}^{2}}}{f_{c}}\right)
$$

where $f_{c}$ is the focal length of the camera lens. The refracted angle, $\gamma$, is given by Snell's Law as

$$
\gamma=\arcsin \left(\frac{\sin \beta}{n}\right)
$$

where $n$ is the relative index of refraction. From $\gamma$ the refracted ray now defines a line $\vec{d}$ in the camera coordinate-system where

$$
\begin{gathered}
\vec{d}=d_{x} \hat{i}+d_{y} \hat{j}+d_{z} \hat{k} \\
d_{x}=\frac{\sqrt{d_{y}^{2}+d_{z}^{2}}}{\tan (\gamma)}
\end{gathered}
$$


To determine the range specified by a point in the image plane, the intersection of the line with the laser plane must be determined. Transforming to the global coordinate axes using the transformation equations

$$
\begin{aligned}
& x=x_{c} \cos \alpha+y_{c} \sin \alpha \\
& y=y_{c} \cos \alpha-x_{c} \sin \alpha+b
\end{aligned}
$$

the line is now given in the laser frame of reference by the position vector $\vec{r}(t)$ where

$$
\begin{gathered}
\vec{r}(t)=\vec{r}_{o}+\overrightarrow{t d} \\
\vec{r}_{o}=b \hat{j}
\end{gathered}
$$

$b$ is the baseline distance from the camera to the laser and $t$ is a scaling parameter.

The laser plane is defined by the vector $\vec{R}$ where

$$
\begin{gathered}
\vec{N} \cdot \vec{R}=0 \\
\vec{N}=b \hat{j} .
\end{gathered}
$$

$\vec{N}$ is the laser-plane normal vector. The intersection of the ray trace and the laser plane occurs at $\vec{r}\left(t_{i}\right)$, as shown in Figure 3.4, where

$$
t_{i}=\frac{-\vec{N} \cdot \vec{r}_{o}}{\vec{N} \cdot \vec{d}}
$$

or substituting (3.22), (3.27), and (3.29) into (3.30)

$$
t_{i}=\frac{-b}{d_{y}}
$$

and (3.22), (3.27), and (3.31) into (3.26)

$$
\vec{r}\left(t_{i}\right)=\left(\frac{-b}{d_{y}}\right)\left(d_{x} \hat{i}+d_{z} \hat{k}\right) .
$$




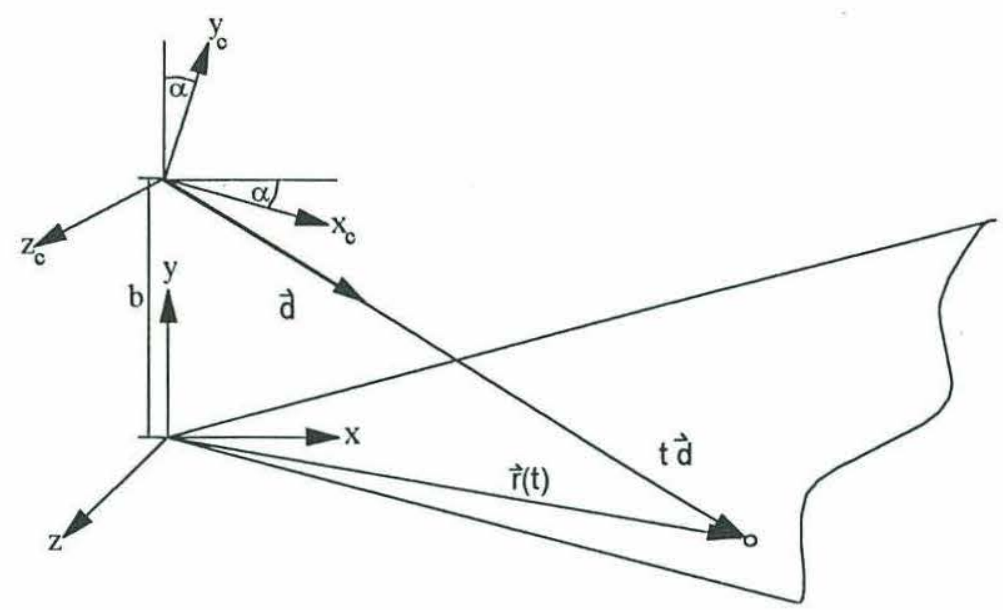

Figure 3.4: Object localization within the laser plane

\subsection{Predicted Range Error}

Range errors incurred by a laser-plane structured-lighting technique include those caused by lens aberrations, image quantization, laser-line localization, and calibration errors in determining camera and laser relative positions. Calibration errors are affected by the method of calibration employed and are difficult to quantify. For this study it is assumed that calibration errors are negligible when compared with other sources.

\subsubsection{Lens-Aberration Error}

In a laser-plane structured-lighting ranging system the image point determines a ray path used to find an object's location within the laser plane. However, in a real camera-lens system the image point corresponds to several possible ray paths due to lens aberrations [27]. The overall effect is that a point on the image plane corresponds to a small disc on the laser-light plane. The effect on range-measurement performance of the system is that an object anywhere within this disc is considered as being at the disc's center. A camera-lens system typically measures the lateral lens aberration (perpendicular to the optical axis) in the image plane of best focus [27]. Results are reported as radial distortion and are a function of the height of ray incidence along the lens. These results are nonlinear and difficult to model. For the purpose of this error analysis the worst-case distortion is assumed for all image 
locations and determined by projecting the distortion onto the laser plane. This is combined in a later section with the other error sources to determine total system range error.

\subsubsection{Laser-Line Localization Error}

As discussed in Section 3.1 the laser light usually illuminates more than a single pixel. Methods to determine which pixel corresponds to the laser-plane center should be implemented to minimize errors from this source. The geometry of a structured-lighting system can determine the number of pixels illuminated by a thin laser beam at varying ranges; divergence will cause its thickness to increase with range and must also be taken into account. A typical Nd:YAG laser beam (as used in the system tested) with a full-angle divergence of $2.2 \mathrm{mrad}$ and a beam width of $1 \mathrm{~mm}$ at $1-\mathrm{m}$ range will have a thickness of $8 \mathrm{~mm}$ at a $4-\mathrm{m}$ range. Because of the small angle this increase can be assumed linear. The beam spreading will causes more pixels to be illuminated at higher ranges, increasing the difficulty in correctly locating the laser line.

\subsubsection{Quantization Error}

To determine ranges, the camera image must be quantized into individual pixels. Each pixel transforms into an area patch on the laser plane as shown in Figure 3.5. The area patch is determined by defining each pixel as a small square on the image plane and transforming its four points as in the previous section. An object that lies within this area patch illuminates the corresponding pixel and the range is determined as that which corresponds to the pixel's center. 


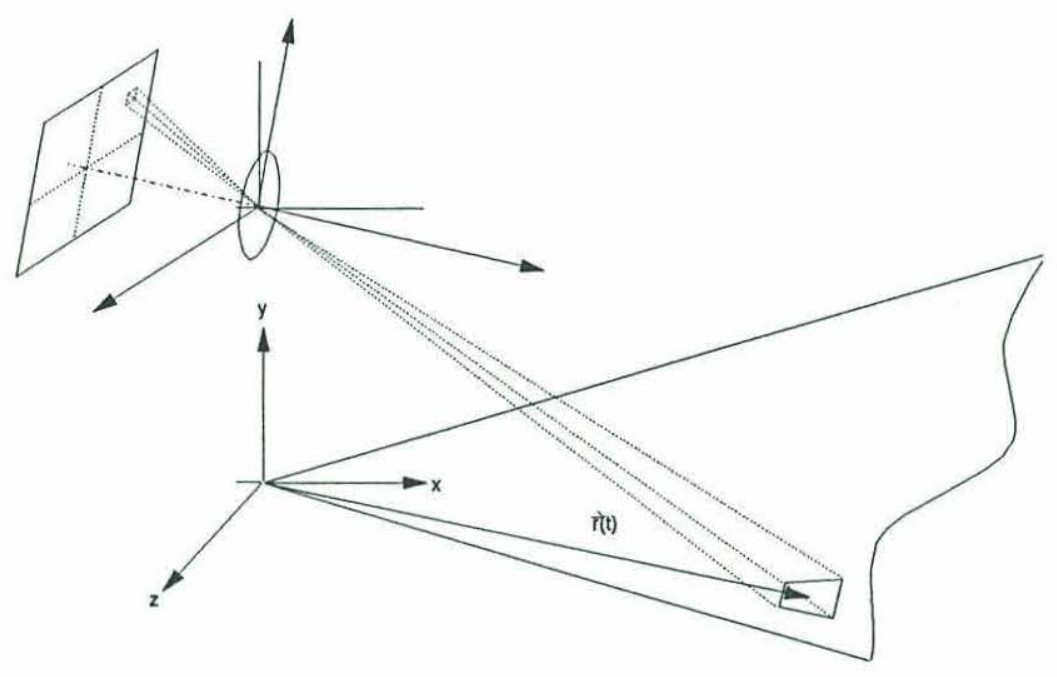

Figure 3.5: Pixel projection on the laser plane

\subsubsection{Total Predicted Range Error}

A laser-plane structured-lighting system, as described in this study, can only determine range to the resolution of the projected pixels. The approach assumes that an object in the laser plane corresponds to a single pixel and reports the center of that pixel as the object's range. An error in the laser-line localization causes a corresponding error in range. These effects are often caused by ambient-lighting noise sources and are difficult to quantify. The range error is predicated by determining all possible ranges that could illuminate a particular pixel. The lens-aberration distortion is included in this range-error determination by adding the maximum distortion size to the pixel boundary then projecting the resultant area to the laser plane. Although the range error occurs in both the $x$ and $z$ directions, the total range error from the laser origin is considered for performance analysis.

The maximum range of an object determined to be at $\vec{r}\left(t_{i}\right)$ corresponds to the distant corner of the area patch shown in Figure 3.5 and the minimum to the near corner. The range error for a particular pixel is defined as the difference between the maximum possible range of an object and the determined range (since the difference between the minimum possible range and the determined range is less because of the geometry). 
Defining the pixel width, including the lens-aberration distortion, as $w$, the maximum range is given by

$$
\vec{r}_{\max }=\frac{-b}{d_{y}-w / 2}\left[\left(d_{x}+\Delta d_{x}\right) \hat{i}+\left(d_{z}-w / 2\right) \hat{k}\right]
$$

where $\Delta d_{x}$ is determined as in Section 3.2 by first computing the new incident angle $\beta_{w / 2}$, which accounts for the pixel-width changes in $d_{y}$ and $d_{z}$ and the new refracted angle $\lambda_{w / 2}$,

$$
\begin{gathered}
\beta_{w / 2}=\arctan \left(\frac{\sqrt{\left(d_{y}-w / 2\right)^{2}+\left(d_{z}-w / 2\right)^{2}}}{f_{c}}\right) \\
\gamma_{w / 2}=\arcsin \left(\frac{\sin \beta_{w / 2}}{n}\right) \\
d_{x}+\Delta d_{x}=\frac{\sqrt{\left(d_{y}-w / 2\right)^{2}+\left(d_{z}-w / 2\right)^{2}}}{\tan \left(\gamma_{w / 2}\right)}
\end{gathered}
$$

This method does not consider possible angular errors at each pixel, however, these are negligible compared to range errors, which are of more concern in determining system performance. An algorithm was developed to determine error at each determined range using this method. The results for a fully functional commercially available system, the SPOTSCAN 2D, are shown in Figure 3.6. The baseline distance $b$ is $150 \mathrm{~mm}$ and the camera angle $\alpha$ is $10^{\circ}$.

From the geometry presented it can be seen that decreases in range error are made possible by increasing the baseline distance $b$. However, as the baseline is increased the transmission path of the light ray is also increased and attenuation loses are correspondingly greater. To demonstrate the sole effect of increasing baseline, Figure 3.7 shows the results for baselines from $100 \mathrm{~mm}$ to $250 \mathrm{~mm}$ with a camera angle of $10^{\circ}$. It should be noted that changing the camera angle has no effect on the range-error performance and only affects the field of view and usable range of the system. 


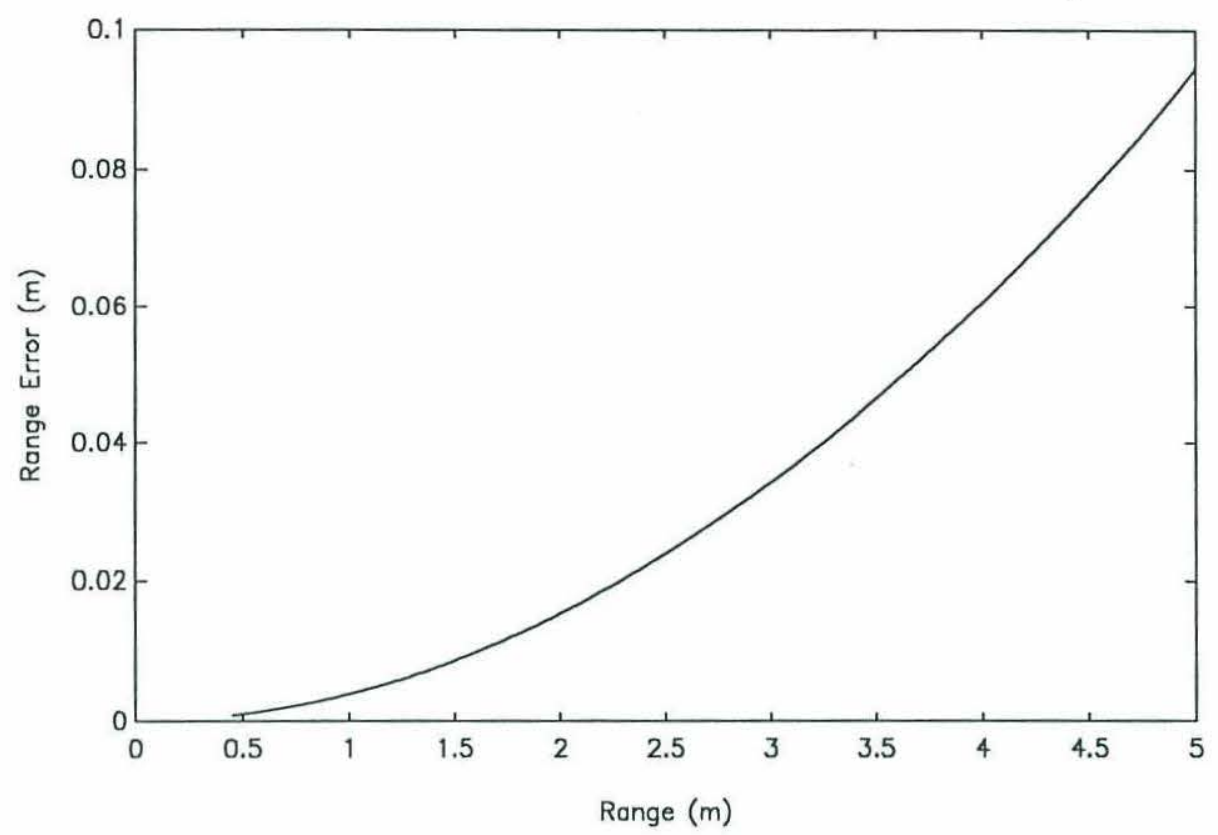

Figure 3.6: Predicted range error for SPOTSCAN 2D

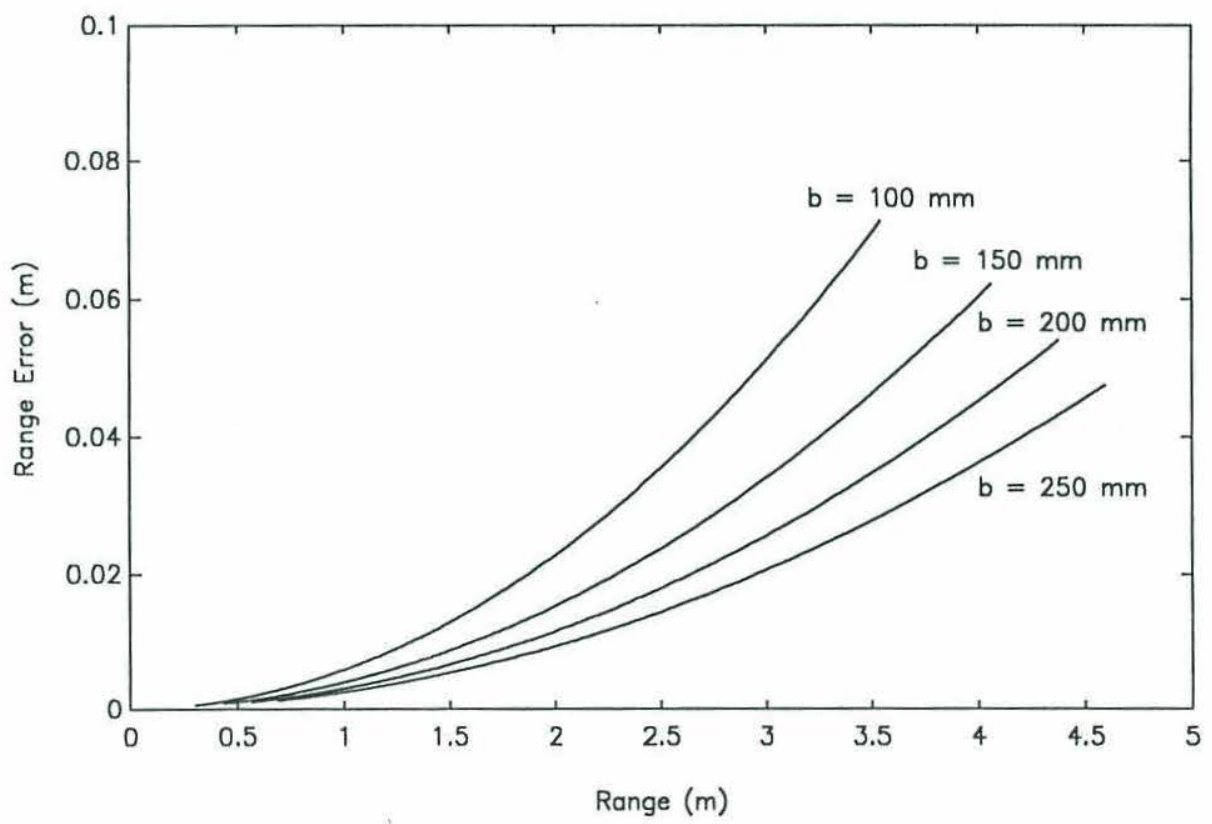

Figure 3.7: Range error for varying baseline 


\subsection{The SPOTSCAN 2D Optical-Ranging System}

The SPOTSCAN 2D system is a fully functional underwater-optical range-finding system employing the laser-camera structured-lighting method, designed and marketed for subsea-pipeline inspection. The system comprises a subsea unit, a scan-processor unit, and a high-resolution-graphics display (Figure 3.8).

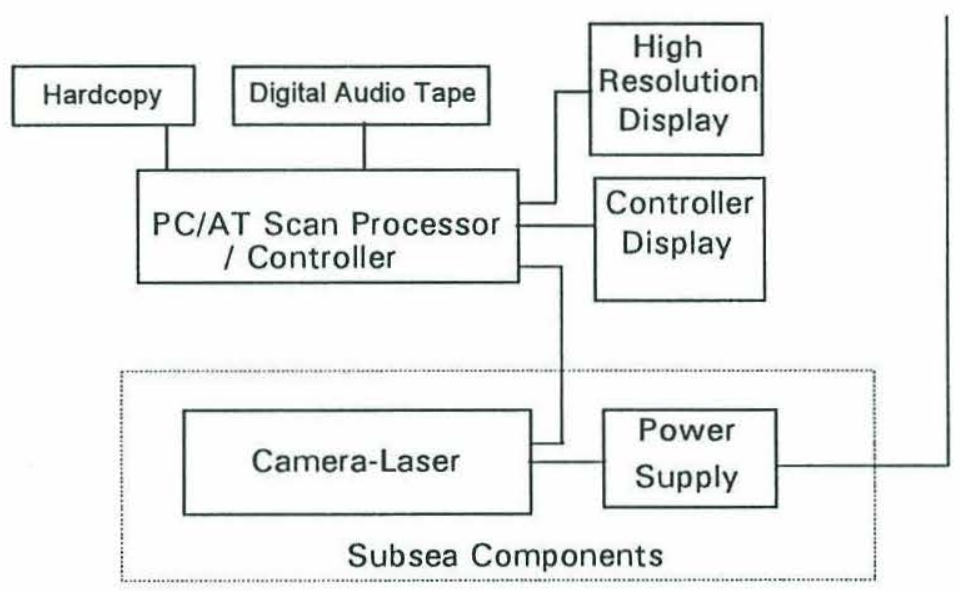

Figure 3.8: SPOTSCAN 2D block diagram

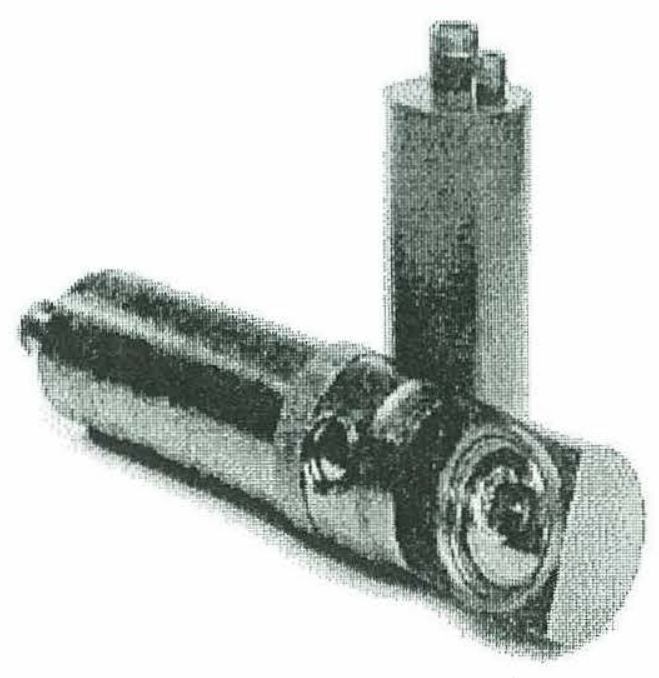

Figure 3.9: SPOTSCAN 2D subsea components 
The subsea unit (Figure 3.9) consists of a camera-laser unit and a laser power-supply unit. The camera-laser unit houses the CCD camera, the laser, and a microprocessor. The camera is designed to detect low light levels and uses automatic gain and iris controls to avoid blooming caused by overexposure [28]. The laser is a 5-mW frequency-doubled, diode-pumped Nd:YAG green laser $(532 \mathrm{~nm})$. A humidity sensor to detect leakage and inclinometers for pitch and roll measurements are also contained within the camera-laser assembly. The subsea microprocessor is used for camera control and communications with the surface processor. The laser power supply contains a regulation system to ensure a stable laser-light level. The standard housing is rated for a maximum depth of $1000 \mathrm{~m}$ [9].

Images are digitized and processed by the scan-processor unit using transputer coprocessor technology to obtain real-time display of profile data. The digitized images have a frame resolution of $256(\mathrm{H}) \times 192(\mathrm{~V})$ and the CCD dimensions are $14.8 \mathrm{~mm} \times 11.1 \mathrm{~mm}$. The camera system uses a wide-angle lens with a focal length of $25 \mathrm{~mm}$. The CCD system-control software runs on a $80286 \mathrm{AT}$ personal computer at $16 \mathrm{MHz}$ and is displayed on a separate monochrome monitor; profiles are displayed on the secondary high-resolution graphics monitor. Data can be stored on digital audio tape (DAT) secondary storage or directly to the processor unit's disk drive. A hardcopy unit is included to provide documentation of the video display [9] .

The subsea unit is calibrated by the manufacturer using a high-precision three-dimensional range. Calibration parameters are stored and accessed by the processor unit for computing profile data. Pitch and roll measurements are used along with the video frame image to output profile points in a gravitational frame of reference.

Previous testing of the SPOTSCAN 2D has been carried out to demonstrate its use as a pipeline inspection tool [9]. These tests have shown a typical accuracy of $5 \mathrm{~mm}$ at a range of $1 \mathrm{~m}$ and reported a quadratic variation of accuracy with range. Although, in Section 3.3.4 it was shown that range accuracy is based on transcendental functions. 


\section{Chapter 4}

\section{CONTROLLED TESTING}

In the last chapter, predicted range error of the SPOTSCAN 2D laser-ranging system was determined. To verify these expectations the system was tested in a controlled environment with known constraints. The results of those test are presented here with comparison to the analytically determined error.

\subsection{Test Setup}

The laser system was tested in a cylindrical tank of depth $3.3 \mathrm{~m}$ and diameter $4.4 \mathrm{~m}$. The tank was filled with clear fresh water at approximately $25^{\circ} \mathrm{C}$. Figure 2.1 shows that attenuation of the frequency-doubled Nd:YAG blue-green laser is minimal for these test conditions. The effects of water clarity, salinity, pressure, and temperature were not tested as part of this study.

A $20-\mathrm{cm}$ diameter pipe section was placed at the tank bottom and used as a primary target. The subsea unit was suspended and positioned at different ranges from approximately $1.0 \mathrm{~m}$ to $3.3 \mathrm{~m}$ from the target's center. Positioning of the pipe at the bottom of the tank close to the tank wall also allowed the tank itself to be used as a target. The tank walls actually became crucial in determining system performance since their perpendicularity could be assumed, making system position determination easier. For the close-range test, at approximately $0.6 \mathrm{~m}$ from the bottom corner of the tank, an additional target was used. A cylindrical shape (a screwdriver handle) of approximate $25-\mathrm{mm}$ diameter was found near the tank's corner. Two data sets were taken at this range: one with the pipe as the primary target and the other using the small cylinder. 


\subsection{Data}

\subsubsection{Data Acquisition}

At each range, approximately 75 profiles were recorded on the DAT recorder at intervals of $1 \mathrm{~s}$. Prior to profile recording the system position was allowed to stabilize so that movement of the camera-laser subsystem would be minimal during profile recording. The number of profiles and average ranges to the primary target center and tank's bottom corner are included in Table 4.1 for each of the five data sets.

\begin{tabular}{|c|c|c|c||}
\hline Data Set & Profiles & Primary Target Range & Tank Corner Range \\
\hline & & $(\mathrm{m})$ & $(\mathrm{m})$ \\
\hline 1 & 79 & 0.97 & 1.02 \\
\hline 2 & 73 & 1.01 & 1.27 \\
\hline 3 & 75 & 1.95 & 2.42 \\
\hline 4 & 73 & 2.59 & 3.25 \\
\hline 5 & 72 & 3.27 & 4.05 \\
\hline
\end{tabular}

Table 4.1: Data set average ranges 


\subsubsection{Raw Video Imagery}

The SPOTSCAN 2D system also includes hardware that allows hardcopy output of the raw video signal, however, several seconds are required for each copy and only a single copy was obtained for each data set. Examples of the raw image are shown in Figures 4.1 through 4.3.

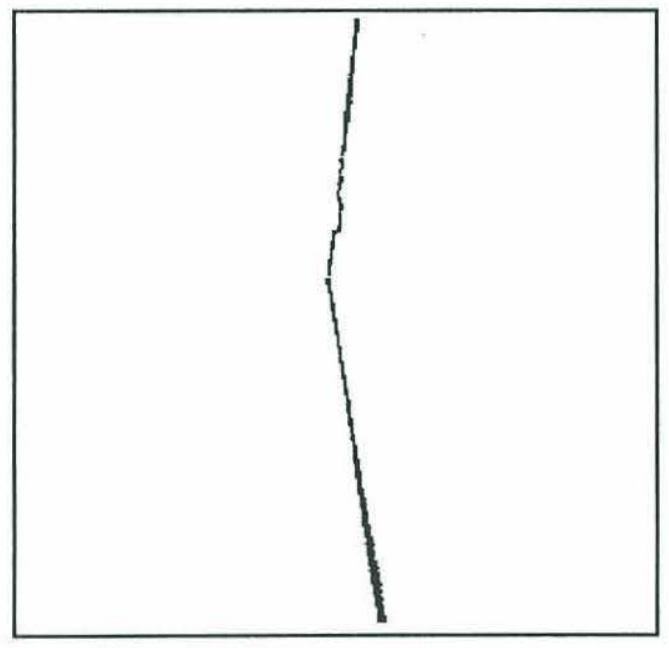

Figure 4.1: Video signal for data set 1

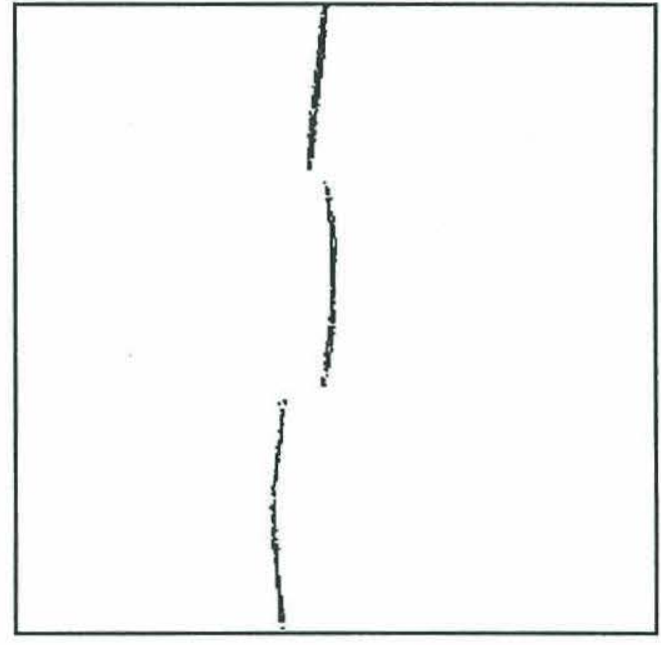

Figure 4.2: Video signal for data set 2

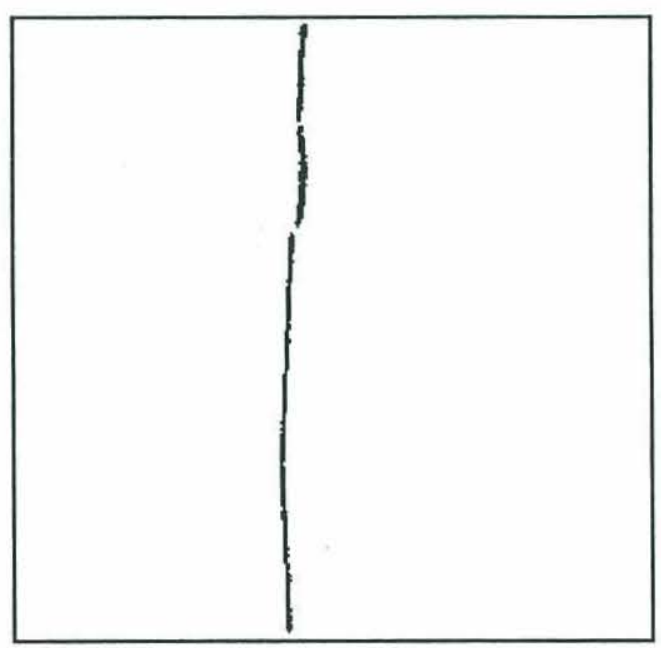

Figure 4.3: Video signal for data set 3 


\subsubsection{Processed Profiles}

The system output consists of up to $256(x, z)$ locations within the laser-plane. The raw video images are processed in a manner similar to that described in Section 3.2. The image is digitized and divided into 256 horizontal lines. For each horizontal line the location of the laser return signal is determined from the center of mass of thresholded image intensity values. Using this location as the $d_{z}$ range of the image and the line number as the $d_{y}$ range, the distances in the laser-plane $x$ and $z$ directions are determined. If the intensity in a line is below a certain threshold, a corresponding point is not determined. Figures 4.4 through 4.8 contain sample processed profiles for data sets 1 through 5 respectively. Individual data points are plotted as small circles for clarity and do not indicate an accuracy range.

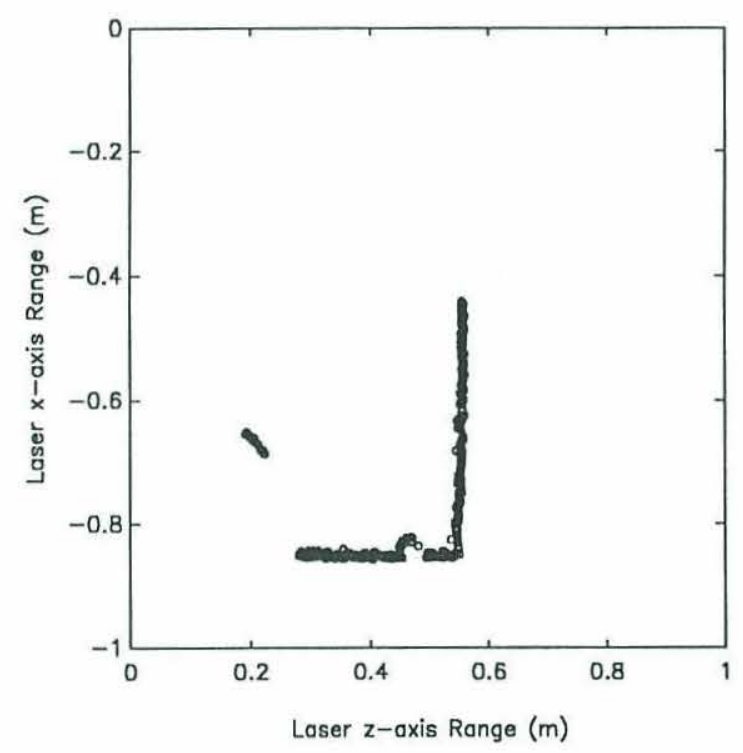

Figure 4.4: Sample profile for data set 1

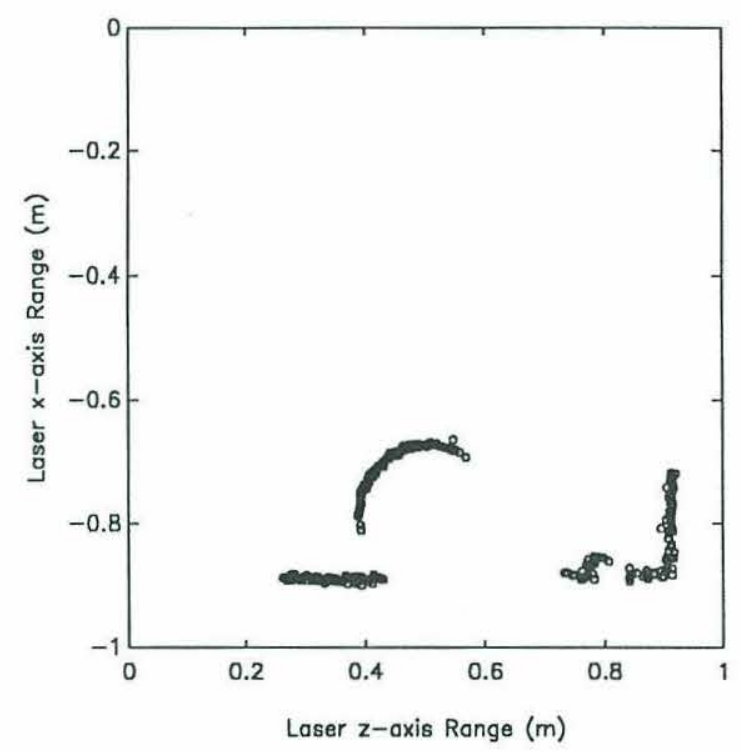

Figure 4.5: Sample profile for data set 2 


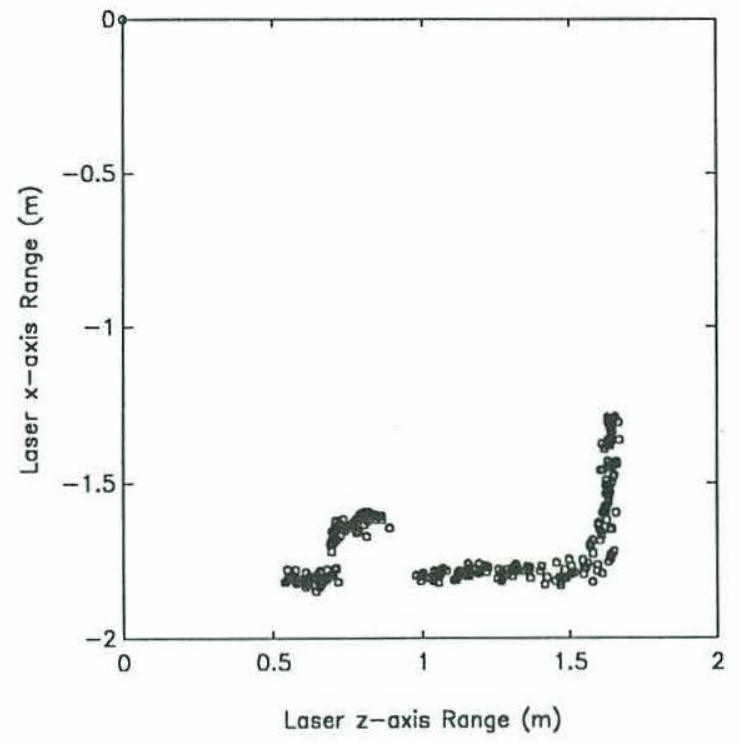

Figure 4.6: Sample profile for data set 3

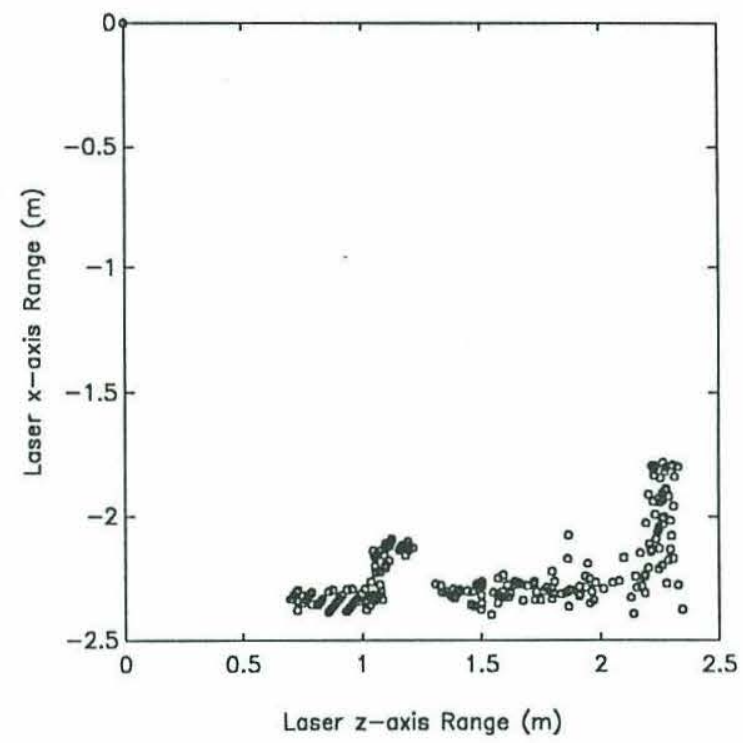

Figure 4.7: Sample profile for data set 4

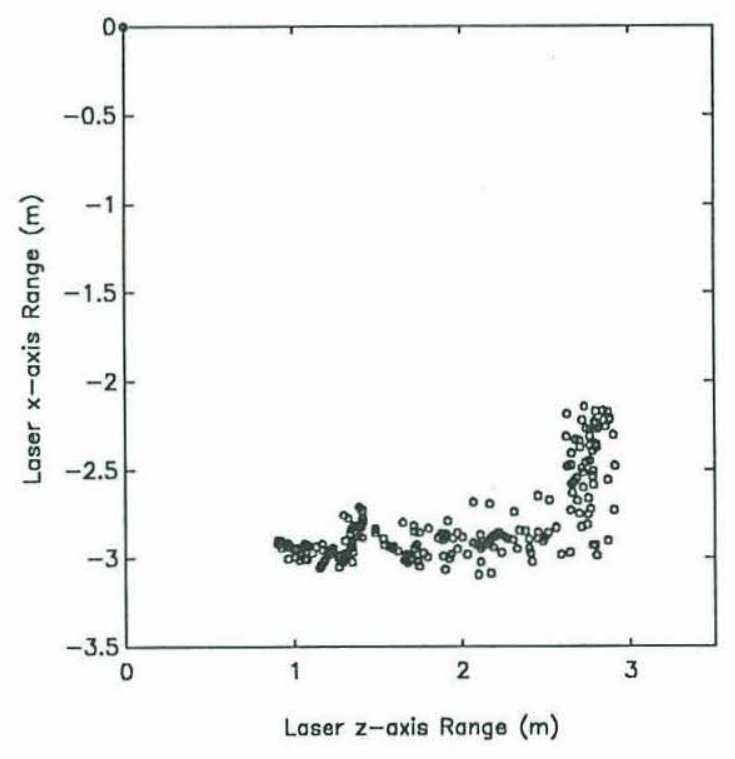

Figure 4.8: Sample profile for data set 5 


\subsection{Range-Error Determination}

Range Error is computed by comparing the laser-system determined range with the actual range to the target. The underwater test made it difficult to measure the actual distances so an alternate method was developed. A model of the tank and primary target is developed for each of the data sets, and the range error between the output data and the model determined. Errors are accumulated for all ranges and the average error used to empirically determine the system's range-error performance

\subsubsection{Tank and Target Model}

The tank model assumes the walls to be exactly straight, horizontal, and vertical. A circular model is used for the primary targets with diameter of $20 \mathrm{~cm}$ for the pipe section and $25 \mathrm{~mm}$ for the smaller cylinder. Varying parameters of the model include the precise wall locations and the primary target location with respect to the tank corner. The wall locations are determined for each individual profile since they can vary slightly due to any small motion of the subsea unit. On the other hand, target location is assumed fixed with respect to the tank's bottom corner throughout each data set since there is no relative motion between the target and tank. Target location with respect to the tank's bottom corner varies between data sets due to repositioning of the subsea unit longitudinally along the pipe.

Horizontal wall location is determined from the average range of points falling within a band in the vicinity of the wall. The assumed error band is from the analytically determined error shown in Figure 3.6. In addition, all points within twice the error distance from the primary target or vertical wall are omitted from the group. Since this method requires knowledge of the vertical wall and primary target locations it must be accomplished iteratively and concurrently with the determination of other parameters. The vertical wall location is found in the same manner using horizontal displacements without regard to the primary target location, since in all cases the target is sufficiently far from the vertical wall.

Target location is determined by first making a visual estimate then correcting each individual profile to minimize the range error of those points obviously on the target. The 
target location used for each data set is then determined by taking the average of the relative distance between the target center and the vertical wall location for each profile.

Table 4.2 contains the average values of the wall locations and the target locations for each data set. Figures 4.9 through 4.13 show sample models used in the range-error determination for each data set.

\begin{tabular}{||c|c|c|c||}
\hline $\begin{array}{c}\text { Data } \\
\text { Set }\end{array}$ & $\begin{array}{c}\text { Average Horizontal } \\
\text { Wall Location } \\
(\mathrm{m})\end{array}$ & $\begin{array}{c}\text { Average Vertical } \\
\text { Wall Location } \\
(\mathrm{m})\end{array}$ & $\begin{array}{c}\text { Primary Target } \\
\text { Displacement from } \\
\text { Vertical Wall }(\mathrm{m})\end{array}$ \\
\hline 1 & 0.55 & 0.85 & 0.09 \\
\hline 2 & 0.91 & 0.89 & 0.41 \\
\hline 3 & 1.63 & 1.79 & 0.83 \\
\hline 4 & 2.26 & 2.34 & 1.11 \\
\hline 5 & 2.77 & 2.96 & 1.35 \\
\hline \hline
\end{tabular}

Table 4.2: Model parameters 


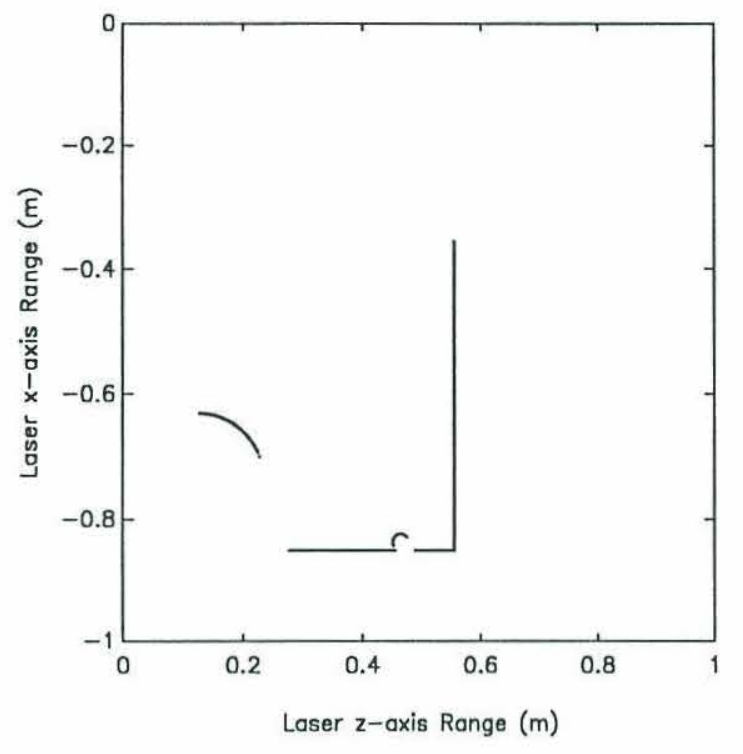

Figure 4.9: Data set 1 model

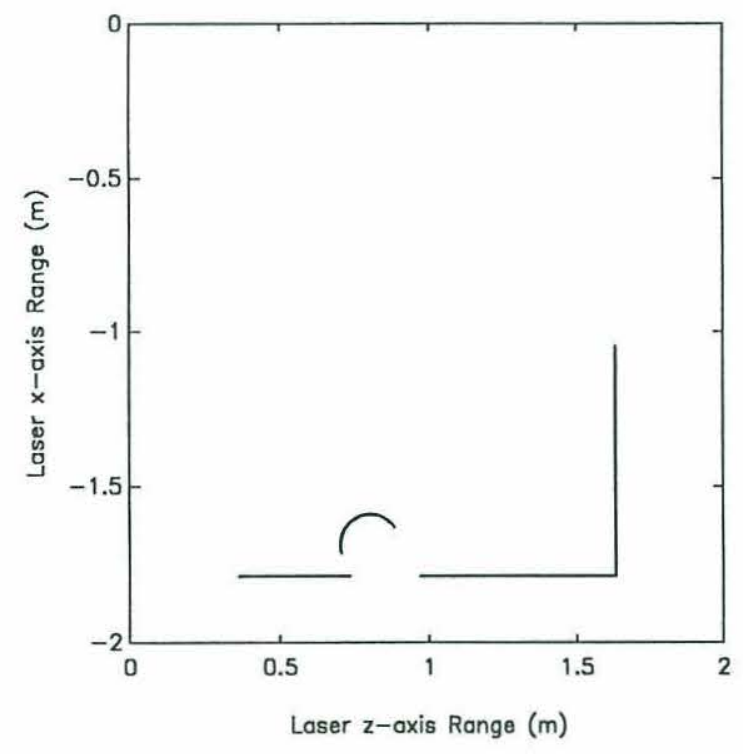

Figure 4.11: Data set 3 model

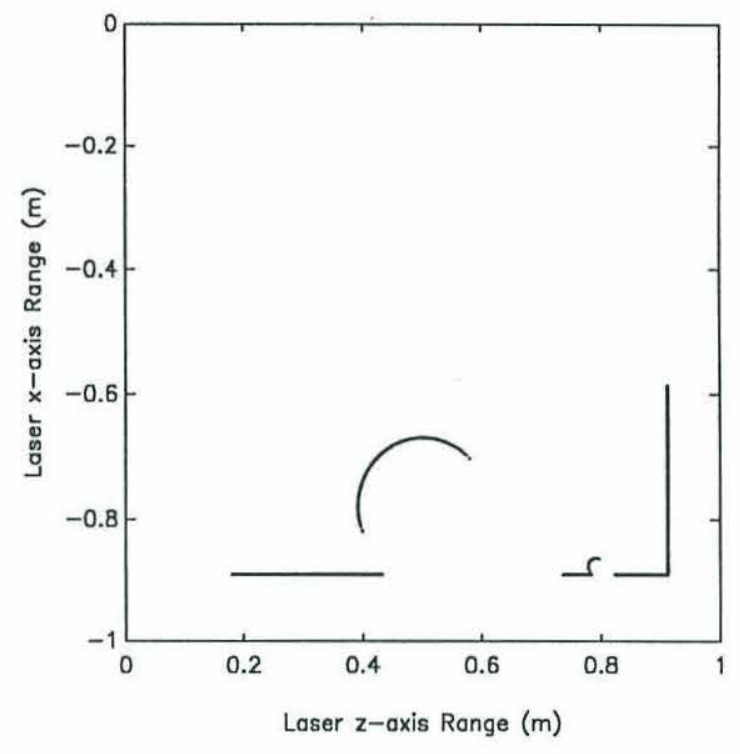

Figure 4.10: Data set 2 model

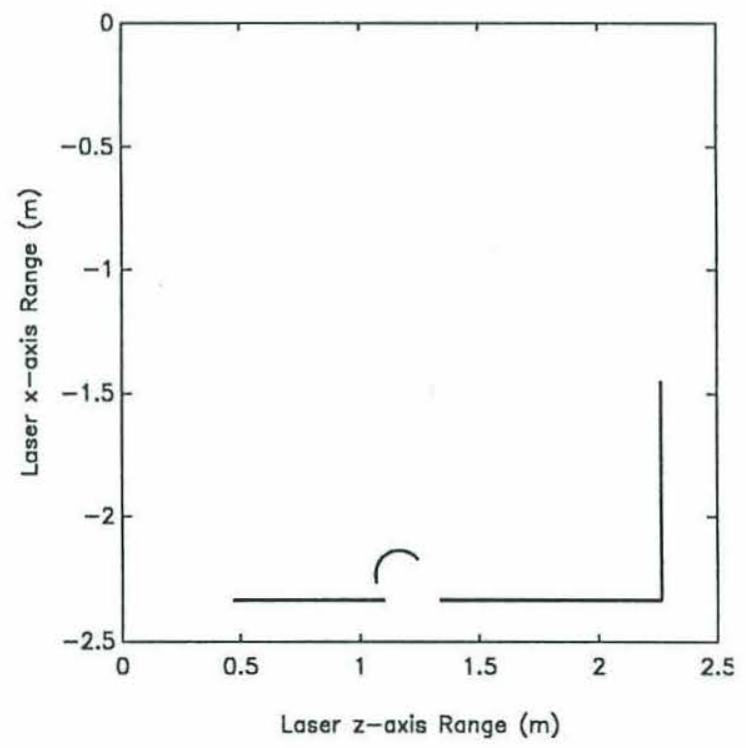

Figure 4.12: Data set 4 model 


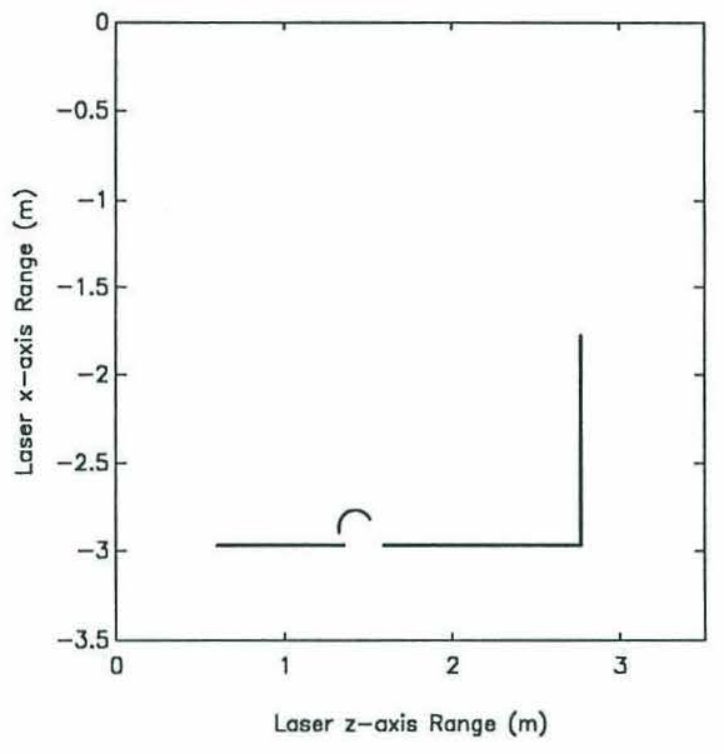

Figure 4.13: Data set 5 model

\subsubsection{Range-Error Computation}

The range-error computation consists of comparing the laser system's determined range at each data point to the range of the model. For each data point a line is drawn from the laser origin through the data point. The range error is computed by determining the distance along this line from the data point to the model. This error is then accumulated in bins for each range using the range to the model as the true range. These accumulated errors are then averaged for each range bin to determine the total range error shown in Figure 4.14. To ensure statistical significance only bins with 100 or more accumulated range-error values are included in the plot. 


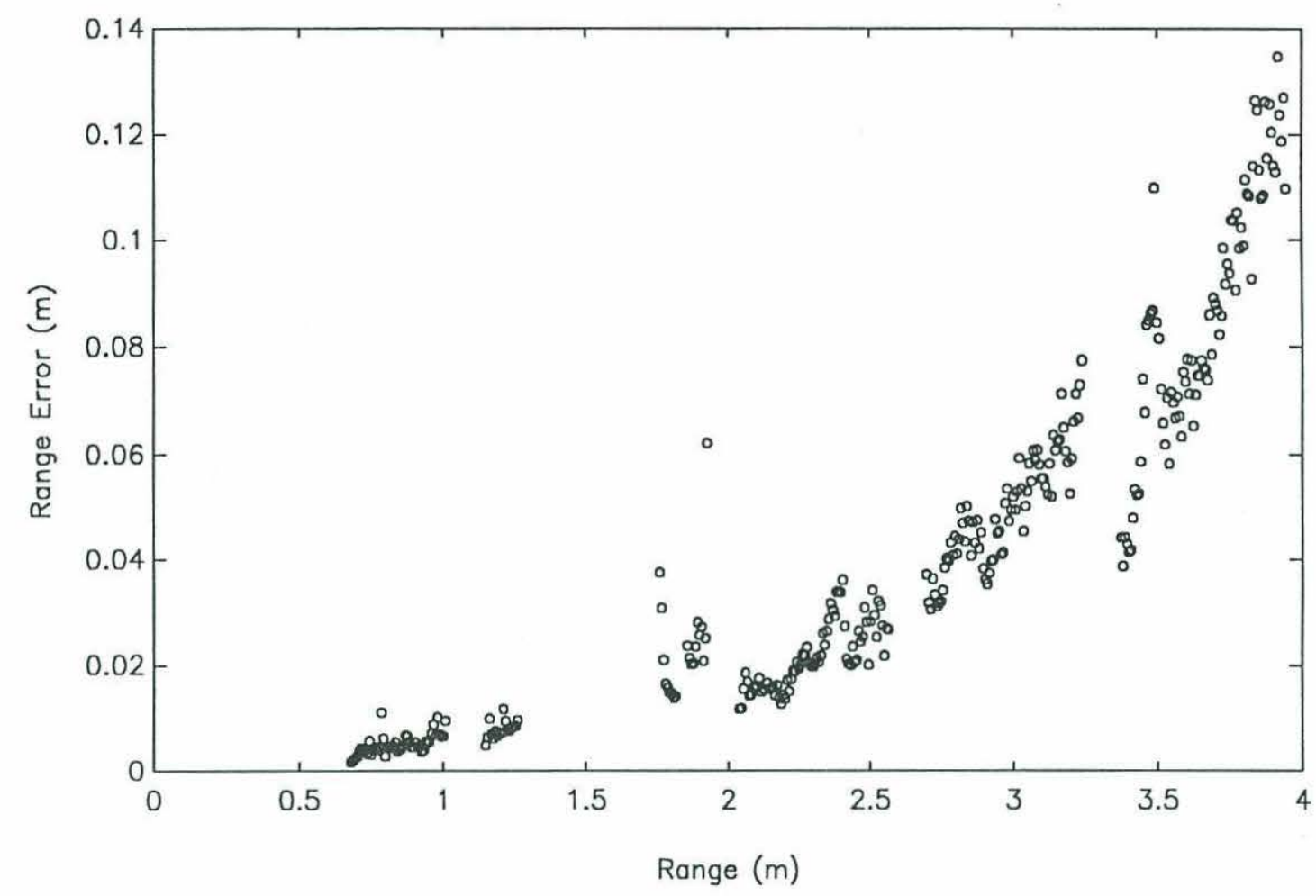

Figure 4.14: Observed range error

\subsection{Empirical Results}

The shape of the data plotted in Figure 4.14 suggest a quadratic curve fit [9]. Using a least-squares method the following empirical relationship is derived for range error $e_{r}$ in terms of range $r$ :

$$
e_{r}=a_{0}+a_{1} r+a_{2} r^{2}
$$

Another selection for an empirical relationship can be obtained by making second-order approximations to the method of Section 3.2. First, consider that the ray path to the laser plane makes an angle $\phi$ with the $y$ axis of Figure 3.4. Then consider that the angular variation of the refracted ray (obtained by moving from pixel to pixel) can be 
approximated by a constant $\Delta \phi$. The range $r$ to the object in the laser plane is given in terms of the baseline $b$ and the angle $\phi$ by

$$
r=b \tan \phi
$$

and the change in range $\Delta r$ from moving to the next pixel is given by

$$
\Delta r=b(\tan (\phi+\Delta \phi)-\tan \phi)
$$

or

$$
\Delta r=\frac{b \sin \Delta \phi}{\cos \phi \cos (\phi+\Delta \phi)}
$$

For ranges greater than $1 \mathrm{~m}$ with a baseline $b$ of $0.15 \mathrm{~m}$, the angle $\phi$ is greater than $81.5^{\circ}$ and a small-angle approximation is feasible for the opposing angle $90^{\circ}-\phi$. Now writing $\Delta r$ as

$$
\Delta r=\frac{b \sin \Delta \phi}{\sin \left(\frac{\pi}{2}-\phi\right) \sin \left(\frac{\pi}{2}-\phi-\Delta \phi\right)}
$$

or with a small-angle approximation

$$
\Delta r=\frac{b \Delta \phi}{\left(\frac{\pi}{2}-\phi\right)\left(\frac{\pi}{2}-\phi-\Delta \phi\right)}
$$

This can be written as

$$
e_{r}=\frac{1}{a_{0}+a_{1} r+a_{2} r^{2}}
$$

since with the small-angle approximation $\phi$ is linearly proportional to $r$ and all other terms are constants. Using this approximation, a least-squares curve fit is non-linear and difficult to calculate. However, by taking the reciprocal of the above equation the problem is greatly simplified. The results from this fit and the previous direct quadratic fit are shown in Figure 4.15 along with the analytical results of Section 3.3. 


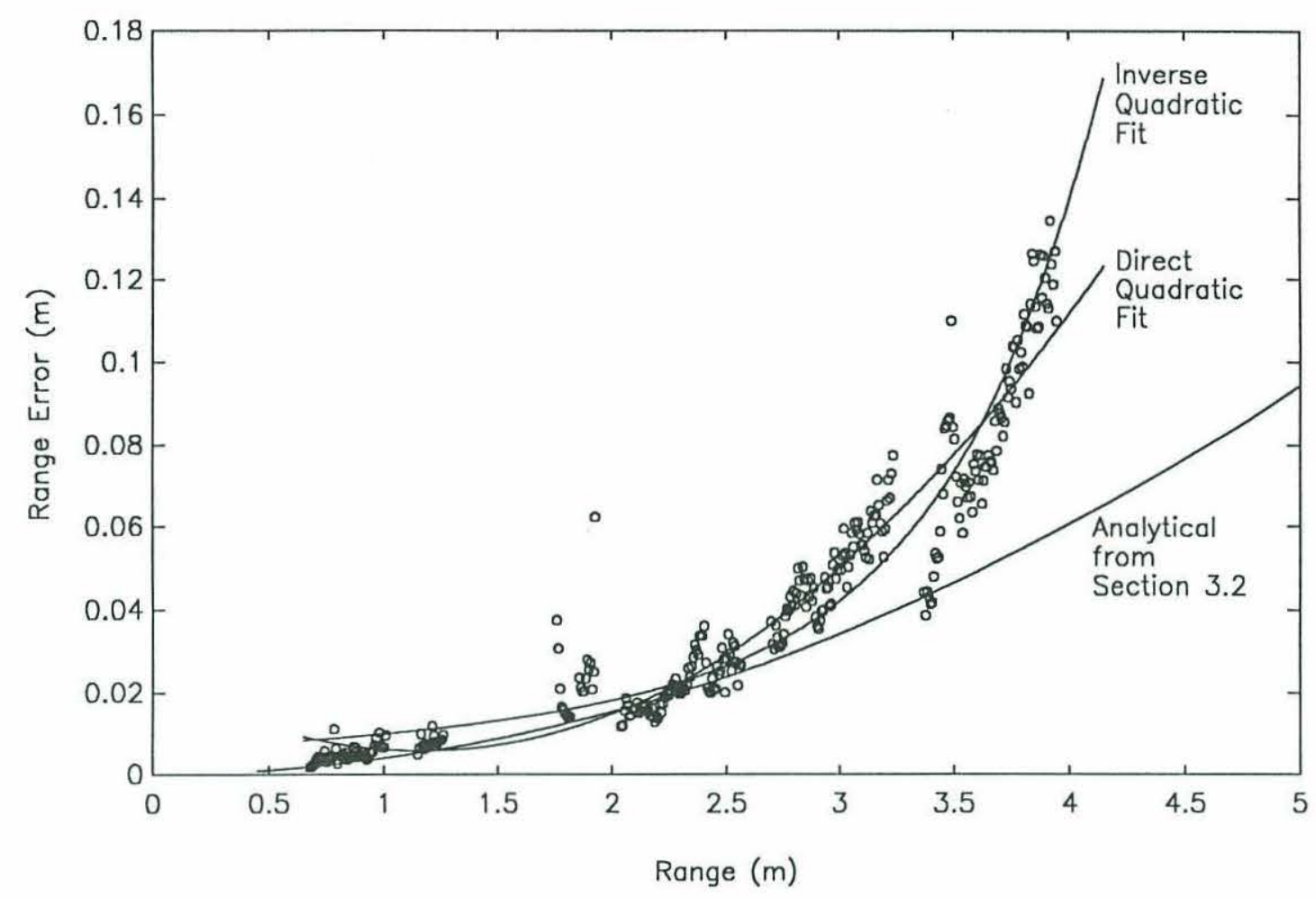

Figure 4.15: Empirical and analytical results

\subsection{Comparison of Empirical and Analytical Error}

Figure 4.14 shows the range error determined by controlled testing of the SPOTSCAN 2D laser-ranging system. These tests confirm previous reports of 5-mm accuracy at 1-m range [9]. Significant error begins to occur beyond $2.5 \mathrm{~m}$. At approximately $3.5 \mathrm{~m}$ there is a distinct break in the trend of the error. The range-error values in this area are entirely from data set 5 and suggest an anomaly associated with these data alone, however, no plausible explanation has been found.

Figure 4.15 demonstrates that the analytical error determined in Section 3.2 is very similar to the observed error for ranges less than $2.5 \mathrm{~m}$. However, at the higher ranges the analytical error is less than the observed error by almost a factor of two. This is partly 
attributed to spreading of the image at higher ranges and additional signal loss from attenuation. As the image intensity decreases with range, the auto-iris and auto-gain features attempt to maintain detection by increasing camera exposure. Overexposure produces blooming of the CCD [28], which causes the imaged laser-line to smear and blur. In addition, the error analysis does not consider other sources of error, such as those encountered during system calibration, or errors in the model determinations of Section 4.3.1. 


\section{Chapter 5}

\section{PRACTICAL APPLICATIONS}

Although the SPOTSCAN 2D system has demonstrated commercial success as a tool for subsea pipe inspection an aim of this thesis is to evaluate its feasibility as an instrument for scientific applications. For this study, a high-resolution seafloor survey application was undertaken to investigate the power-law relationship of the seafloor in very high spatial-frequency regions $\left(>10^{1}\right.$ cycles $\left./ \mathrm{m}\right)$.

\subsection{Deep-Sea Modifications}

The SPOTSCAN 2D system as tested in Chapter 4 is rated for depths to $1000 \mathrm{~m}$, which is adequate for the purpose of pipeline inspection. However, for this application full-ocean-depth $(6000 \mathrm{~m})$ capability was required. Figure 5.1 shows the more capable configuration as designed and implemented by the Woods Hole Oceanographic Institution's Deep Submergence Lab and Seatex A/S. Most obvious is the dual-bottle configuration to house the camera and laser subsea unit. An added benefit of this approach is the larger baseline permitted by housing the camera and laser in separate containers. Although vehicle space considerations, system geometry, and laser attenuation prevented a dramatic change, the baseline was nevertheless increased from $150 \mathrm{~mm}$ to $400 \mathrm{~mm}$. This should have improved system range-error performance as discussed in Section 3.2, however, there was no opportunity for controlled testing of this configuration as described in Chapter 4 for the single-bottle. Aside from the housing modifications all other aspects of the system are as described in Section 3.3 and as tested in Chapter 4. 


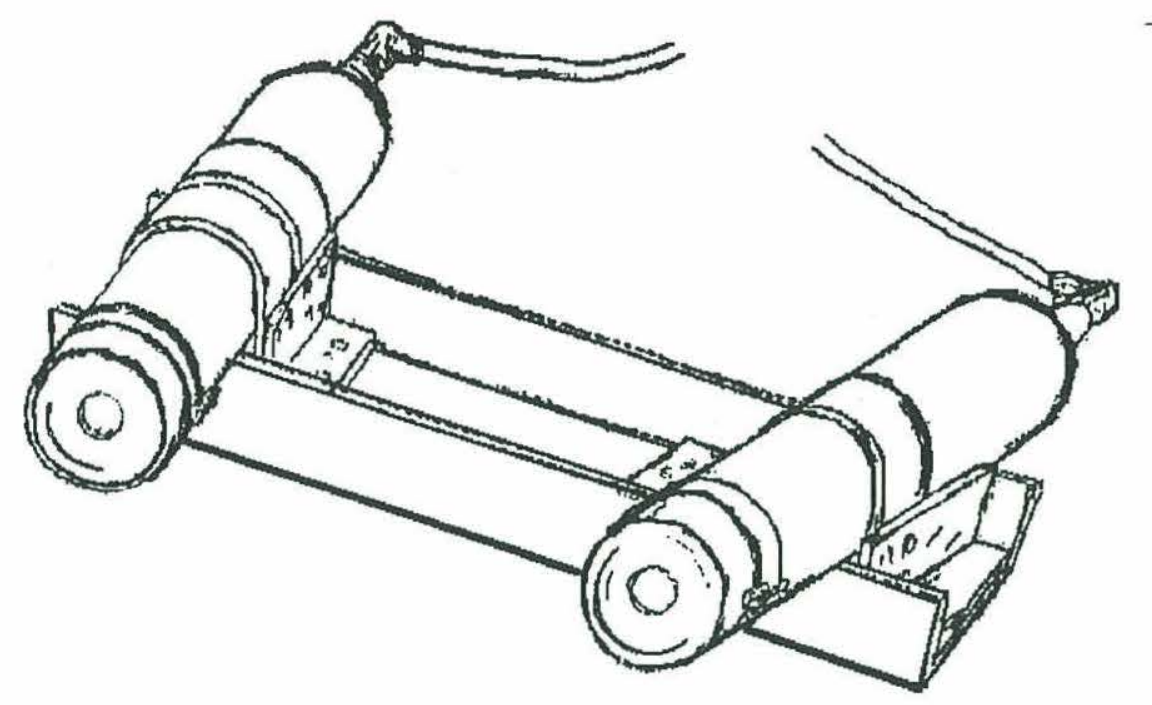

Figure 5.1 : Full-ocean-depth (6000-m) subsea unit

\subsection{The SPOTSCAN 2D System at Sea}

The SPOTSCAN 2D system was employed as a high-resolution survey instrument on the remotely operated vehicle (ROV) Jason (Figure 5.2). The Jason ROV is a full-ocean-depth tethered vehicle capable of performing remote sensor surveys at close ranges with a suite of sonars, video imaging equipment, and electronic still cameras. The vehicle was employed extensively from the R/V Laney-Chouest during the Crest '91 cruise in the Northern Pacific. Project Crest, supported by the Office of Naval Research, included an effort to perform innovative high-resolution, high-precision mapping, and manipulation activities in a challenging ridge-crest environment (2200-m to 4800-m water depth). The primary area of operation was the Endeavour portion of the Northern Juan de Fuca Ridge in the Northeastern Pacific.

A portion of the high-resolution surveys during Crest ' 91 was dedicated to obtaining detailed information about seafloor roughness in the region. The SPOTSCAN 2D laser-ranging system was suited to these high-resolution surveys but had never been deployed at such depths nor used for a scientific study of seafloor conditions. 


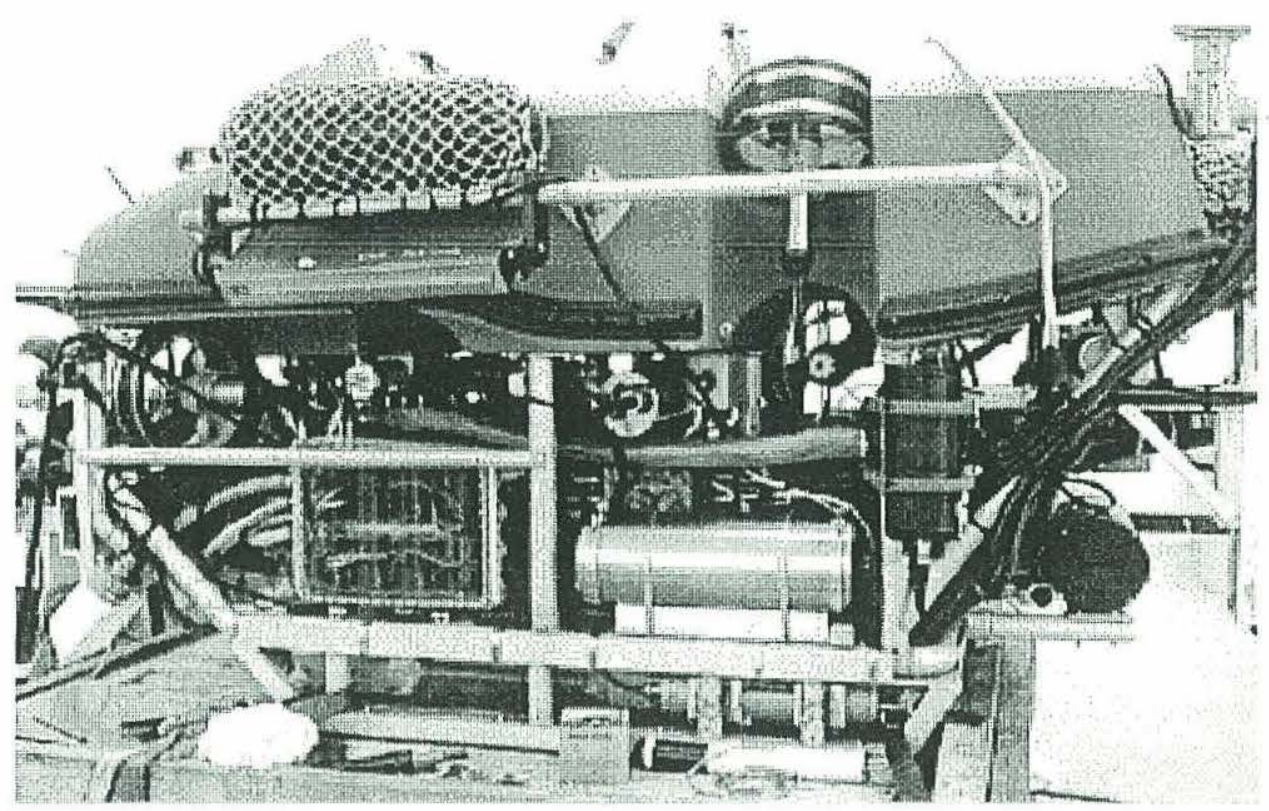

Figure 5.2: Remotely operated vehicle - Jason

The laser system was initially configured in a down-looking mode and mounted aft on the Jason ROV as shown in Figures 5.3 and 5.4. This allowed bottom surveys to be carried out but presented some difficulty due to the limited range of the laser system. Surveys had to be performed at typical altitudes above the seafloor of less than $3 \mathrm{~m}$ for reasonably good profiles (more than 100 data points) because attenuation at longer ranges was too high for a strong light-signal return. In addition, the aft piloting lights of the vehicle had to be secured for optimal laser detection. These affects combined to make piloting of the vehicle an extreme challenge during the high-resolution laser surveys. In order to perform surveys in an alternate configuration, the system was mounted on the forward end of the Jason ROV and in a forward-profiling configuration as shown in Figures 5.5 and 5.6. This mode, combined with the excellent maneuverability of the Jason ROV, allowed detailed surveys to be performed on ledges and escarpments. In this mode the vehicle's piloting lights also had to be secured for laser surveying. The laser system was incapable of functioning to any degree when Jason's forward high-intensity flood lamps were energized. 


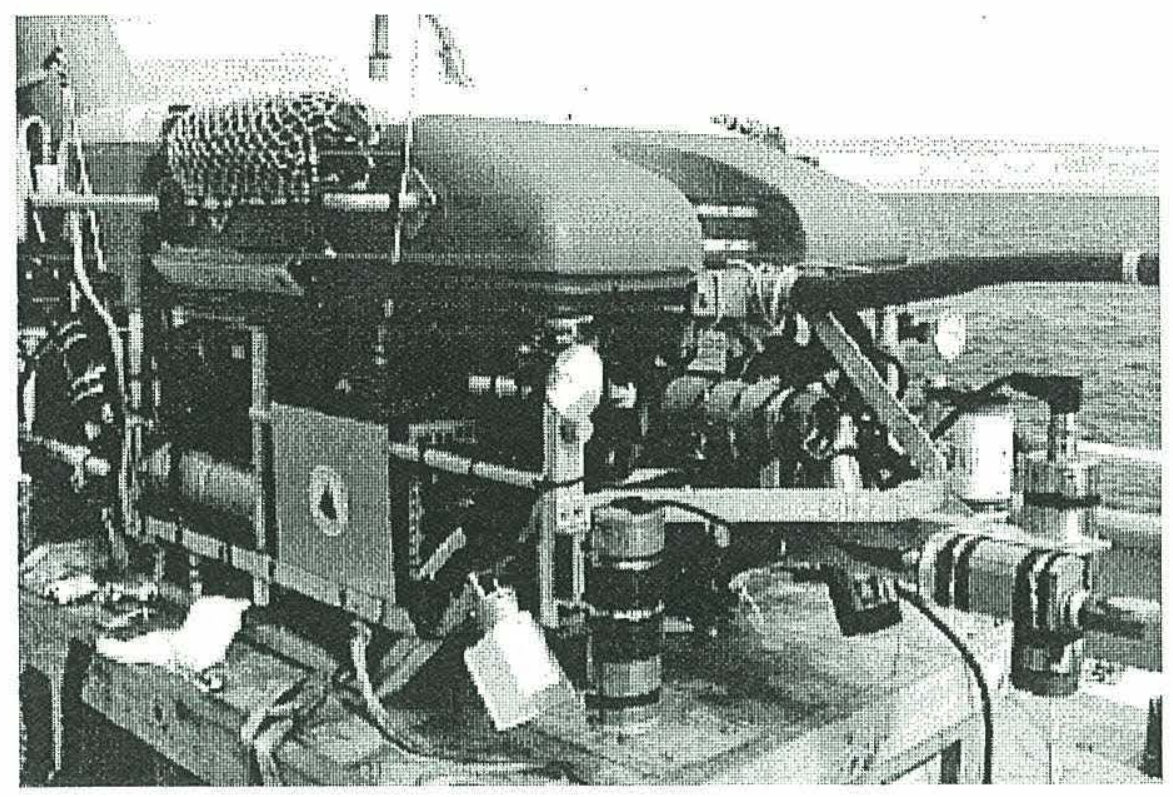

Figure 5.3: Down-looking configuration

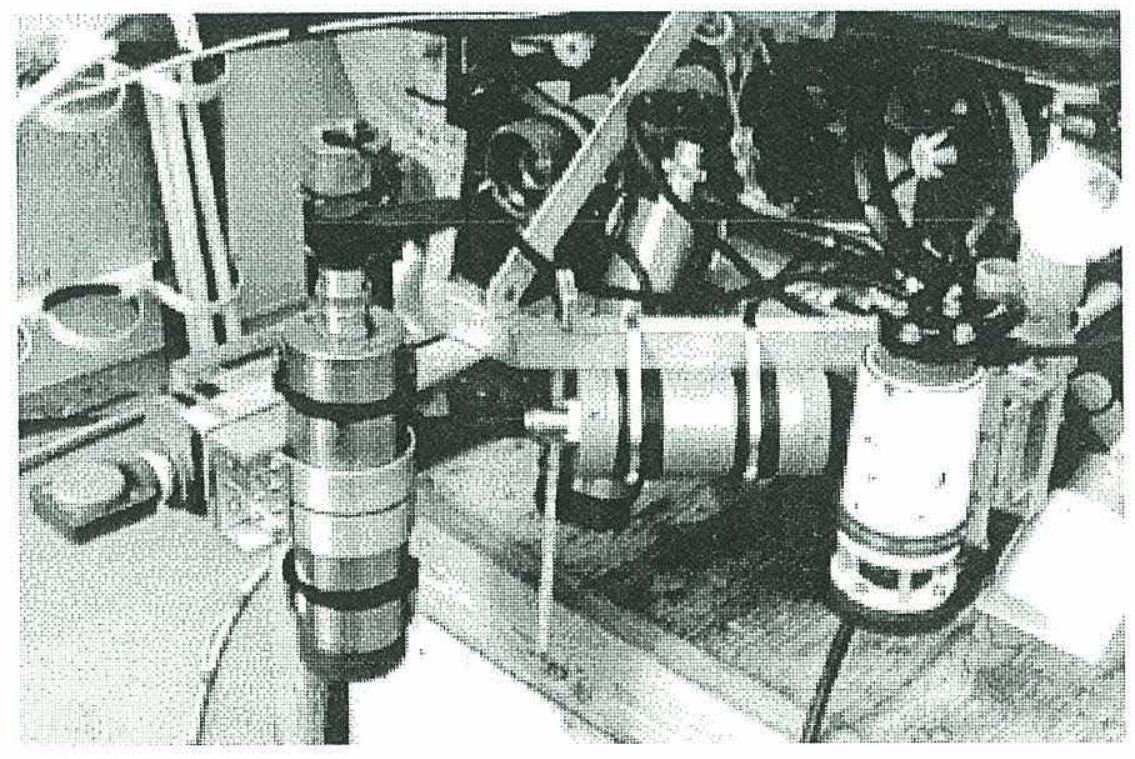

Figure 5.4: Detail of down-looking configuration 


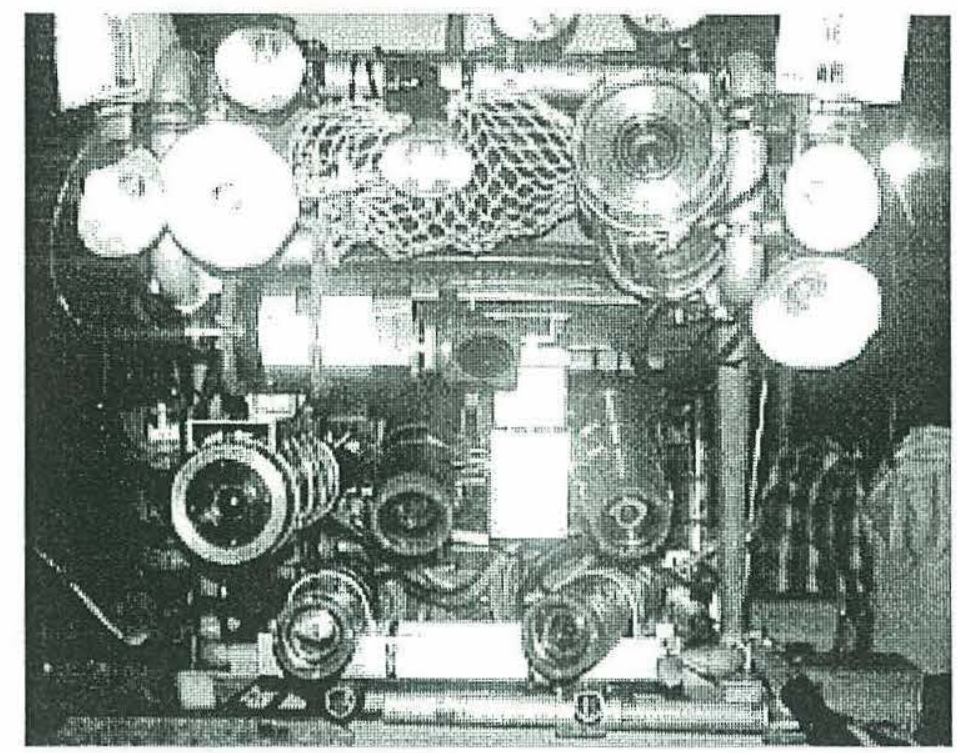

Figure 5.5: Forward-looking configuration

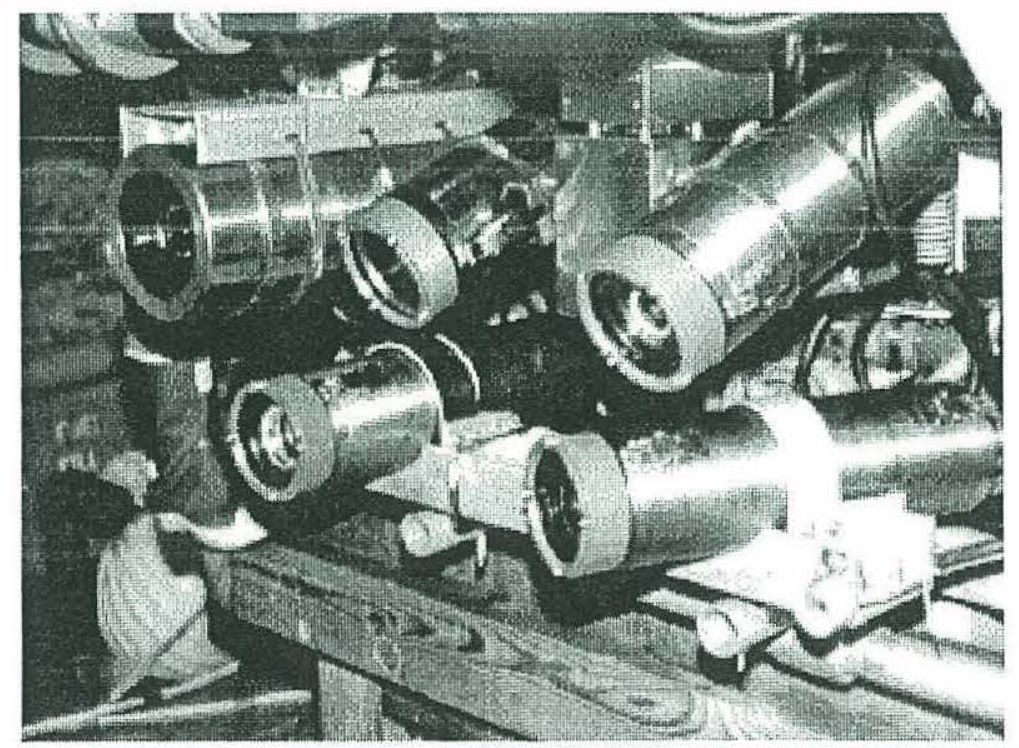

Figure 5.6: Detail of forward-looking configuration 


\subsection{High-Resolution Seafloor Surveys}

Approximately 6600 laser profiles of the seafloor were obtained during the Crest '91 cruise. These can be broken down into roughly 4100 bottom profiles and 2500 forward profiles. The profiles were obtained over two separate 2-day periods on the 4 th and 5 th, and the 15th and 16th of August 1991. Other operations prohibited continuous laser profiling during these periods. Even during the committed laser surveys the difficulties encountered with the ROV piloting lights caused profile recordings to be intermittent. The effect of energized lights corrupted several of the profiles and of the 6600 obtained approximately $50 \%$ have usable data.

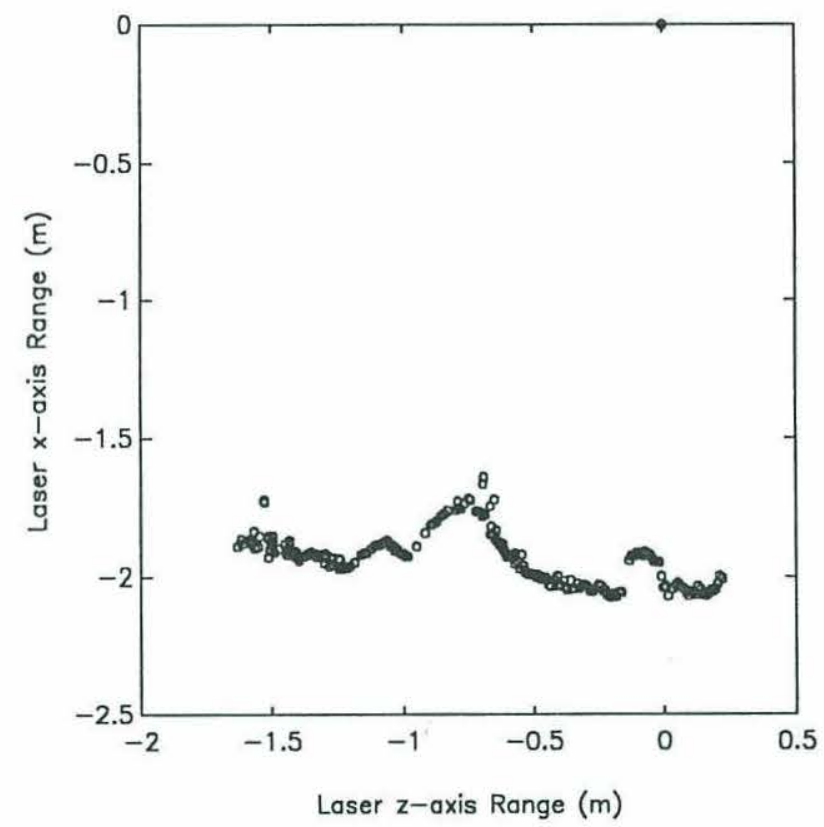

Figure 5.7: Typical bottom profile obtained during Crest ' 91 


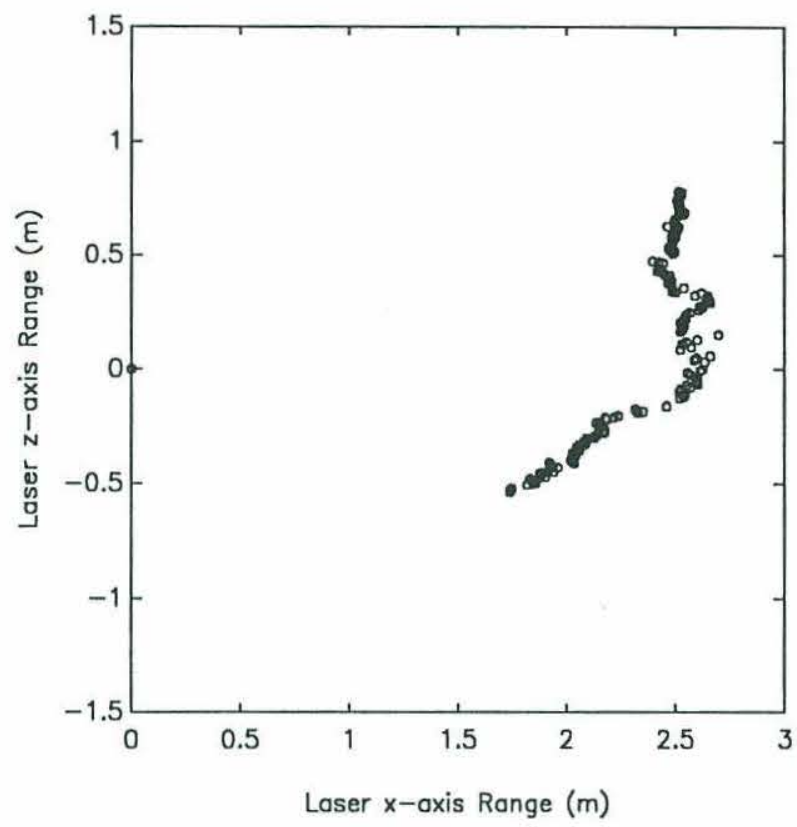

Figure 5.8: Typical forward profile obtained during Crest '91

Figures 5.7 and 5.8 show two typical laser profiles in the down-looking and forward-looking configurations. Under optimal conditions, where all 256 horizontal lines of the image contain intensity values above threshold, the system produces 256 points per profile. Due to the rough and erratic nature of the seafloor this is rarely the case. The uncorrupted profiles had, on average, 200 points in the down-looking configuration and 155 in the forward-looking configuration. The profile of Figure 5.7 contains 210 points and that in Figure 5.8 has 157. In most cases this is sufficient to obtain the necessary seafloor amplitude measurements. 


\subsection{High-Spatial-Frequency Seafloor-Roughness Measurements}

Seafloor-roughness determination has many practical and engineering applications particularly in the field of ocean acoustics [29]. Interactions of acoustic signals with the seafloor are crucial in determining backscatter information. Whether the seafloor acts as a reflector or a scatterer of energy depends on the relative spatial frequencies of the incident acoustic signal and the bottom roughness [2,29]. Accordingly, describing bottom roughness in terms of spatial frequency is necessary and useful in determining these interactions. The determination of high-spatial-frequency seafloor-roughness can be accomplished directly with amplitude measurements, however, due to the immense area of the ocean bottom it is practically impossible to obtain this information globally. In those cases where such measurements have been made, extremely large storage is required for even a small area of high-resolution surveys.

To overcome these limitations some stochastic models have been devised to provide the information statistically rather than deterministically. These models use spectral analysis to describe the seafloor-roughness in terms of the spatial frequency. The model is based on the observation that the power spectral density function of the seafloor profile follows a power law [29] and the log-log relationship of power and spatial frequency is linear. Verification of these stochastic models has relied on high-frequency sonars for spatial frequencies up to $10^{-1}$ cycles $/ \mathrm{m}$ and in the very high range of $10^{2}$ cycles $/ \mathrm{m}$ through the use of stereo-pair bottom photographs [29].

The Crest '91 surveys included high-resolution bathymetric and sidescan sonar surveys along with the laser surveys. The sidescan sonars use frequencies of $120 \mathrm{kHz}$ and $200 \mathrm{kHz}$ and are capable of measuring spatial frequencies of approximately $10^{-1}$ cycles $/ \mathrm{m}$ and $10^{\circ}$ cycles/m respectively. The highest resolution sonar used was a Mesotech scanning sonar operating at $675 \mathrm{kHz}$ and capable of measuring spatial frequencies into the $10^{1}$ cycles $/ \mathrm{m}$ range. The laser system's resolution capabilities of less than $10 \mathrm{~mm}$ permits the measurement of spatial frequencies from $10^{1}$ to $10^{3}$ cycles $/ \mathrm{m}$. 
Current work at the Woods Hole Oceanographic Institution has demonstrated the stochastic model for seafloor-roughness using data obtained from Crest '91. Figure 5.9 shows the approximate power-law relationship of the power spectral density for several sensors employed during Crest '91. The flattening of the $120-\mathrm{kHz}$ curve near $10^{-1}$ cycles $/ \mathrm{m}$ is attributed to white noise at the limit of the instrument's resolving capability. The highest frequencies $\left(10^{3} \mathrm{cycles} / \mathrm{m}\right)$ were obtained with the laser-ranging system. The following section describes power-spectrum determination using laser profiles.

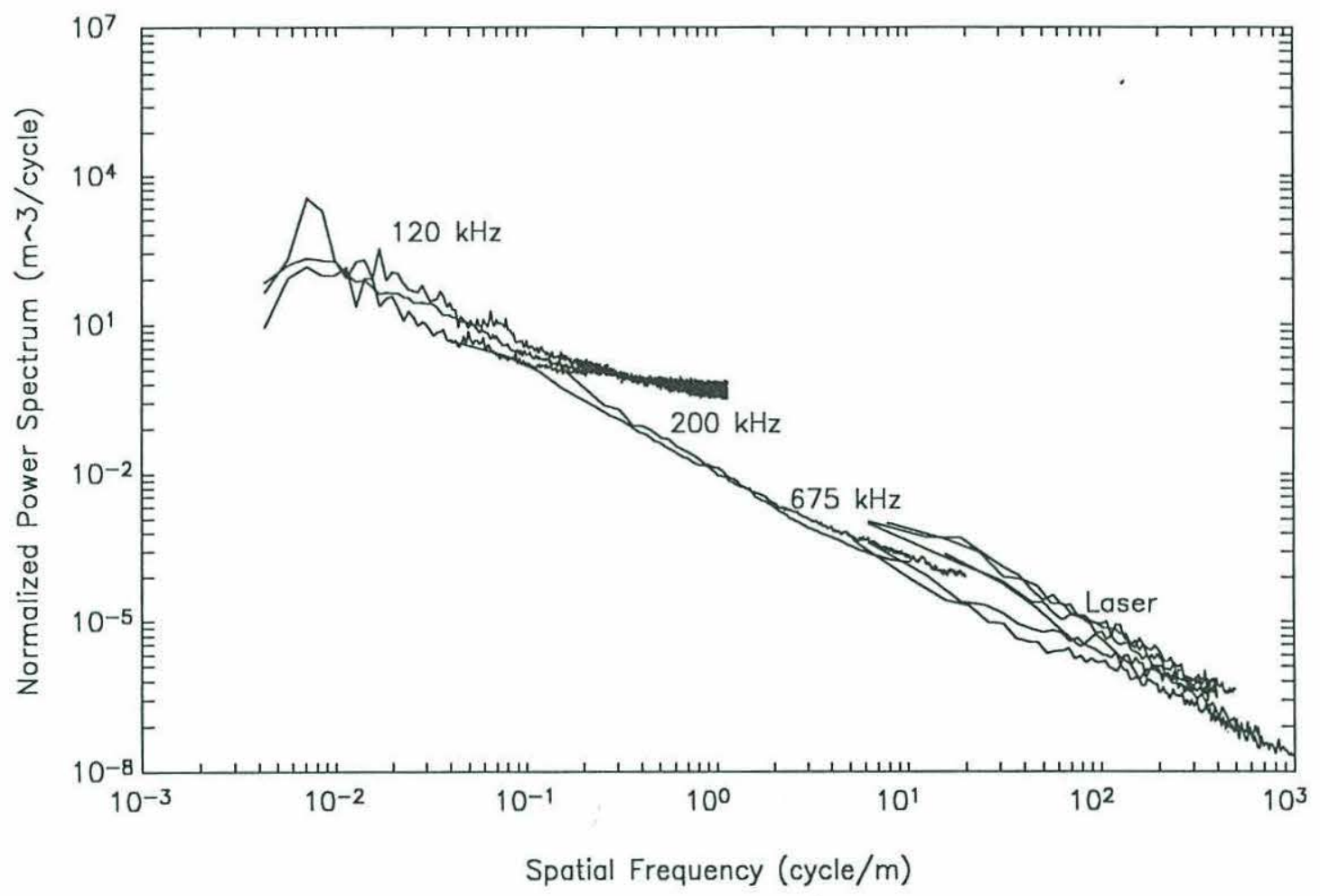

Figure 5.9: Power-law relationship of the seafloor 


\subsection{Power Spectral Density from Laser Profiles}

An approach to determining power spectral density uses the Fourier transform [30]. For real data this normally entails computation of the Discrete Fourier Transform (DFT) or Fast Fourier Transform (FFT) then conversion to the Discrete Fourier Series (DFS), the sampled version of the Fourier Transform [31]. In order to consider the DFS a sampled version of the data's Fourier transform, the data must have an equal sampling interval for all points in the series. The profile points determined by the laser system are not equally spaced and in most circumstances there are one or more gaps in a profile. A solution is to select profiles meeting a criterion that allows the profiles to be resampled at an equal interval with a minimal effect on the representation. Once suitable profiles have been selected and resampled they can be processed using the same algorithm applied to the acoustic data.

\subsubsection{Profile Selection and Resampling}

To smooth the results of the power spectral density function, it is desirable to obtain several profiles from a homogeneous (stationary) area of the seafloor then average the power spectral density for these profiles. To achieve this, data sets with continuous profiling for approximately 10 minutes were chosen. At $1 \mathrm{~s}$ per profile this yields approximately 600 profiles per set. Because of the Jason ROV's slow speeds $(<1 \mathrm{~m} / \mathrm{s})$ it may be assumed the seafloor region of each set is homogenous. Six such data sets were found and used for power-spectrum computation. Each data set is processed by first omitting any profiles that do not contain a minimum number of 128 points; this criterion usually ensures the profile extends over an adequate range and does not have significant gaps. The next step is to determine the average interval of the profile by dividing total profile length by the number of points. The next criterion is to determine if the profile has gaps greater than five times this average interval. If a large gap occurs at either end the profile is shortened to remove the gap and the criterion reapplied. A profile meeting these requirements is considered suitable for resampling. In general, this reduces the number of profiles for a data set to approximately 50 . This is sensitive to the criteria for minimum profile points and gap size but this number was 
sufficient for smoothing the power spectral density function. The high standard of these criteria also ensures that selected profiles are of high quality and yield good results.

Resampling uses a linear interpolation between the data points in a profile. To compute the average power spectral density function from a group of profiles, each profile must have the same sampling interval. If the sampling intervals are not equal, the frequencies corresponding to particular points in two separate series are not the same. Having the same sampling interval allows the individual power spectral density functions to be combined point by point. The sampling interval used is the minimum average interval of the profiles in a data set. The profiles are resampled at this interval for 128 points. This causes all the profiles to have a new length equal to that of the shortest profile in the data set. Figures 5.10, 5.11, and 5.12 show original profiles and the profiles resampled using this method (resampled profiles are offset for clarity). This method ensures that the profiles are not undersampled and that excessively high frequencies are not introduced by the linear interpolation.

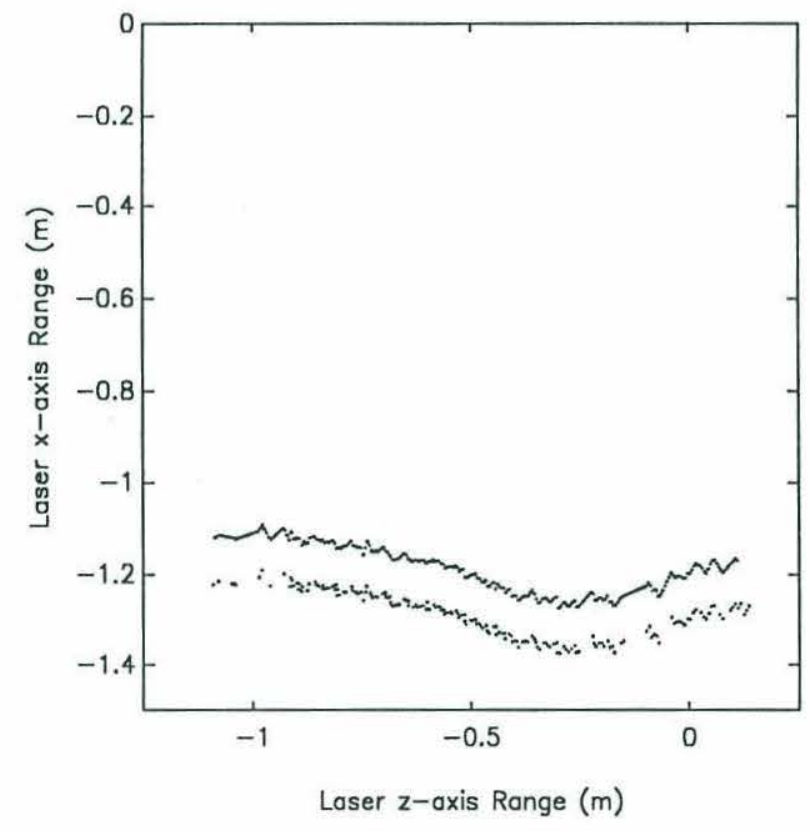

Figure 5.10: Resampled bottom profile 


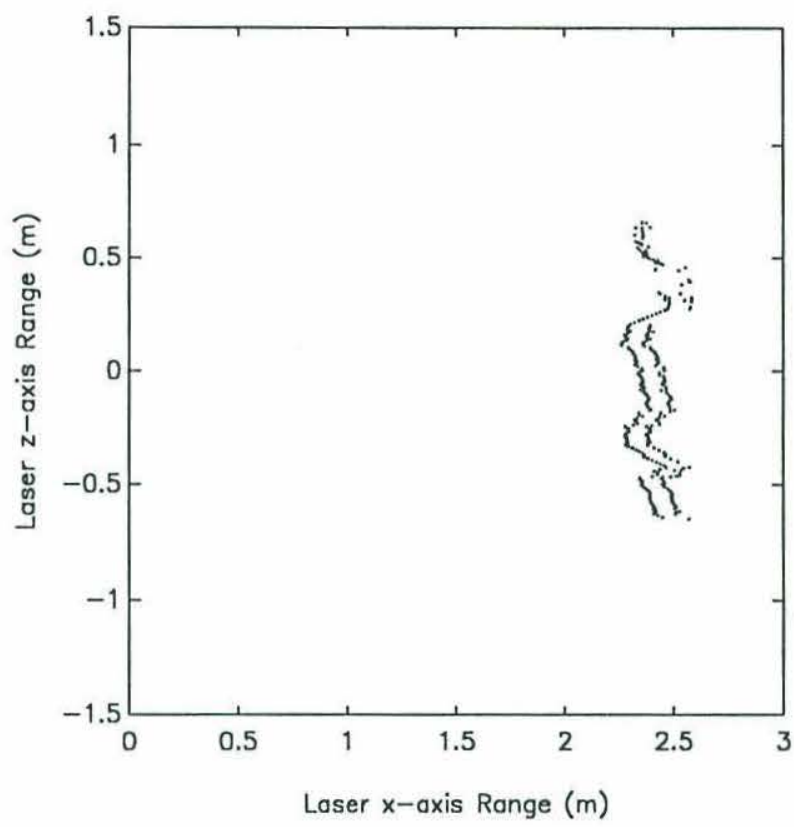

Figure 5.11: Resampled forward profile

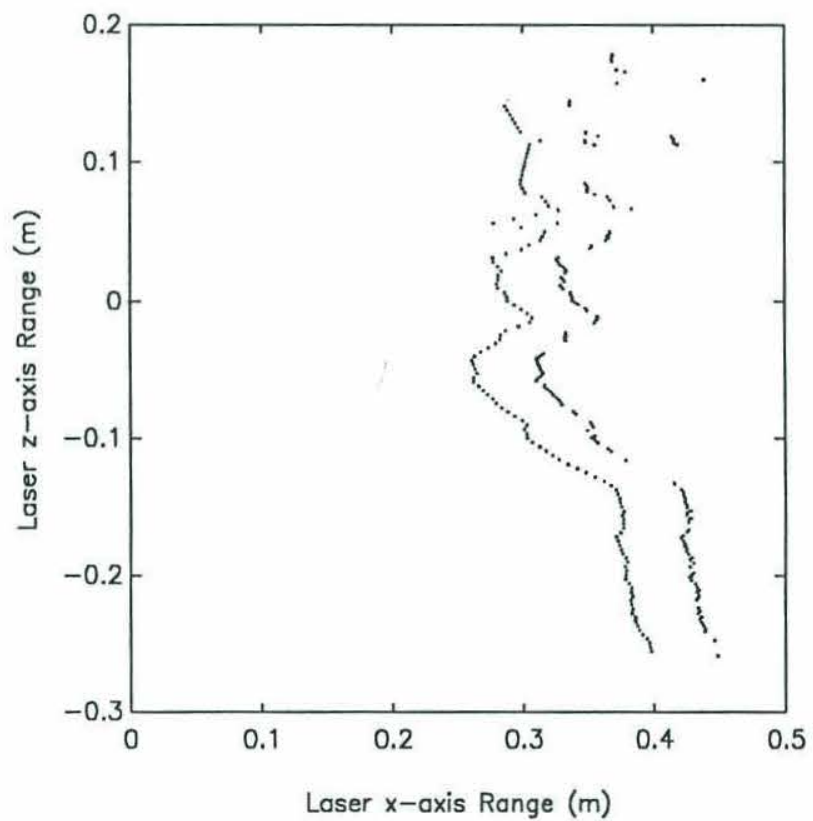

Figure 5.12: Resampled close-range forward profile 


\subsubsection{Power Spectral Density}

The algorithm for determining the normalized power spectral density consists of detrending the profiles to remove low-frequency components to concentrate on the high-frequency roughness measurement. Profiles are tapered on each end using a split Gaussian to reduce aliasing. To determine the power spectral density function, the Fourier transform is computed, multiplied by its conjugate, then normalized by the length of the profile.

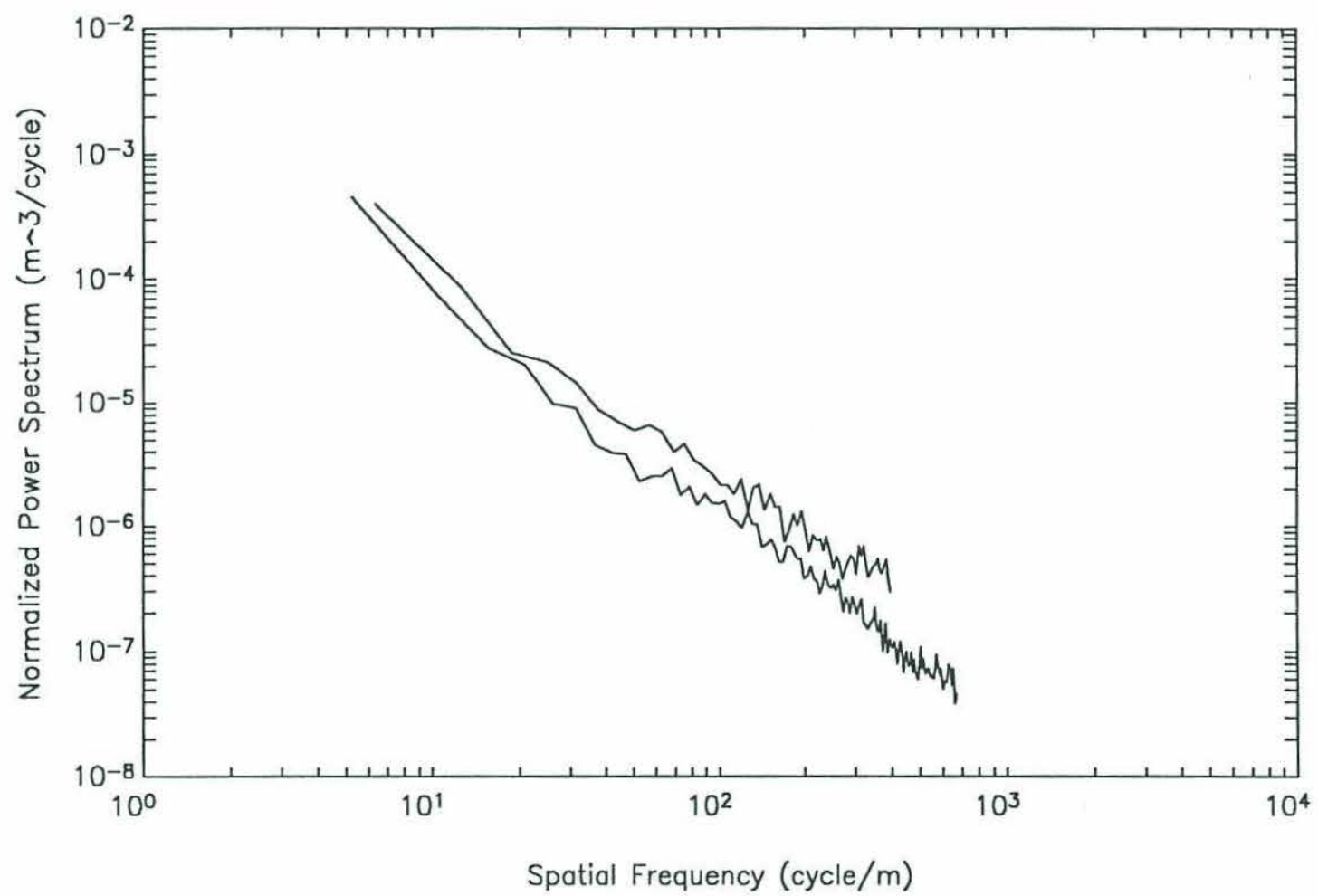

Figure 5.13: Power spectrum of bottom profiles

Figure 5.13 shows the average power spectral density function for two data sets with a down-looking laser configuration. A slightly higher sampling frequency (shorter interval) was used with the second data, which led to the high spatial frequencies shown. Figure 5.14 shows the average power spectral density function for three data sets with the laser in a forward-looking configuration. One data set was collected at an extremely close range (see Figure 5.12), leading to the very high spatial frequencies seen in this plot. Figure 5.15 shows 
all six data sets. As a verification of the power-law theory for seafloor-roughness, the power spectral density function was computed for one data set from the controlled testing in Chapter 4. From the results shown in Figure 5.16 it is clear that the geometric shape of the test tank and target do not produce the same results as the seafloor data.

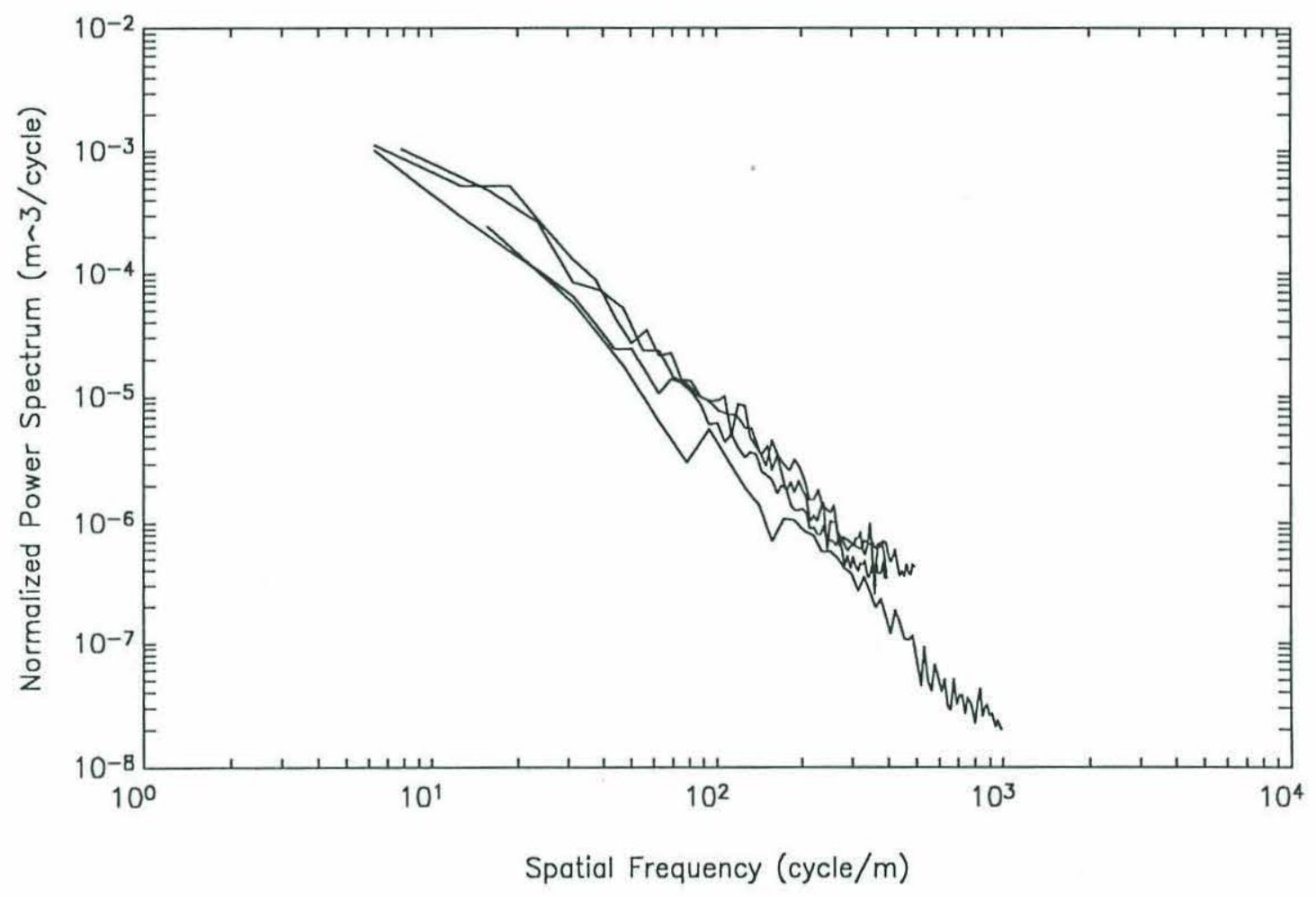

Figure 5.14: Power spectrum of forward profiles 


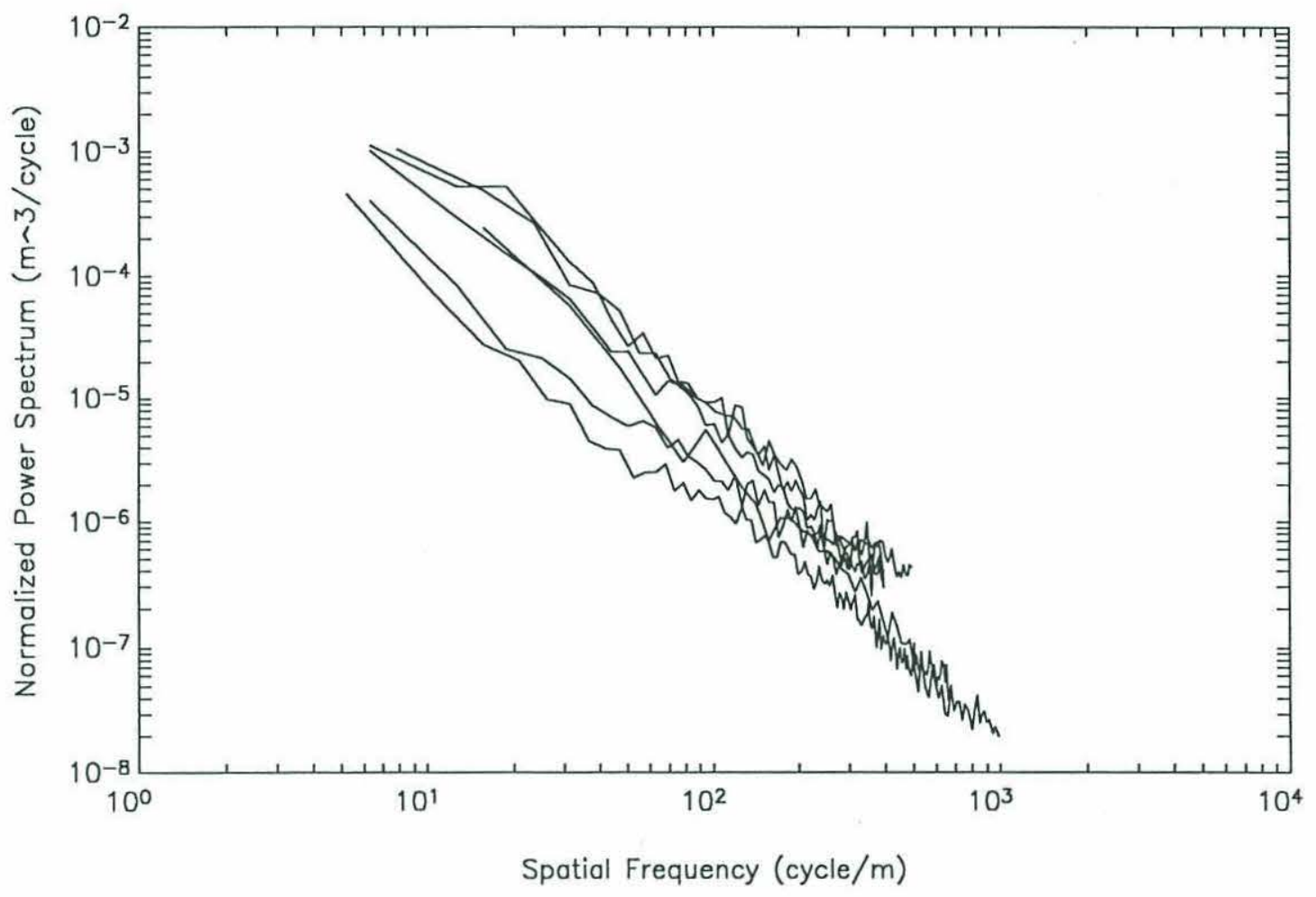

Figure 5.15: Composite power spectrum of laser profiles 


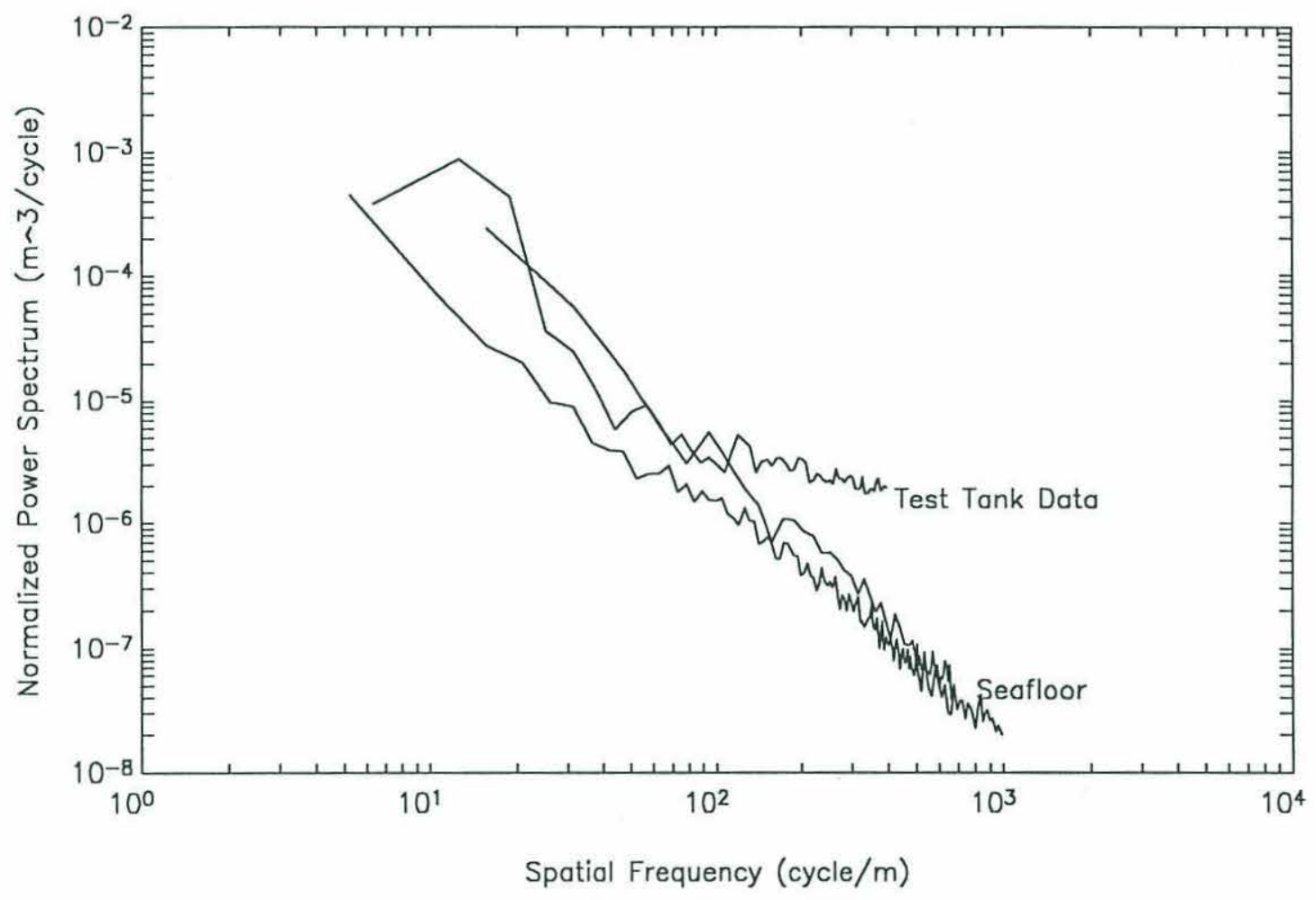

Figure 5.16: Power spectrum of test tank data 


\section{Chapter 6}

\section{CONCLUSIONS}

\subsection{The Practicality of Underwater Optical Ranging}

The drawbacks of traditional acoustic methods for high-resolution measurements in a subsea environment have led to the search for alternative techniques. Successful implementation of in-air optical techniques for the past three decades and recent developments in their employment underwater suggests these methods as a viable option. Three methods of laser ranging have potential in the underwater environment: laser radar, which uses time-of-flight; amplitude-modulation, which employs phase-comparison; and triangulation methods. Advantages of laser-ranging systems are the high angular resolutions possible and the potentially fast survey rates presented by the high velocity of light. A structured-lighting technique using triangulation of a laser plane and a single-camera detection system is chosen because of its simplicity and ease of implementation. A general approach in determining performance of this technique was undertaken and applied to a commercially available system. This system was procured, tested, and applied in an at-sea scientific study to measure seafloor roughness in very high-frequency regions $\left(>10^{2}\right.$ cycles $\left./ \mathrm{m}\right)$. The system is shown to be a feasible and practical option for these very high resolutions $(<10 \mathrm{~mm})$. The laser-range measurements are used to support the approximate power-law relationship of the seafloor power spectral density function and spatial frequency.

The most restrictive limitation of this system is its sensitivity to lighting. In all cases any direct lighting in the system camera's field of view causes complete loss of laser detection. Deployment of the system in the deep-sea environment is performed by mounting on an ROV. Piloting an ROV in the deep-sea typically involves visual imaging and demands extensive lighting. The need to secure this lighting in order to employ the laser ranging system poses severe conditions on piloting. Besides risking damage to the vehicle by flying blindly, staying 
within an acceptable range window is extremely difficult. Corruption of laser profiles due to intermittent use of lights while surveying also imposes additional post processing or the need for hand editing to cull out this data. For this ranging technique to remain a practicable option this limitation must be overcome.

Although an aim of this study is to evaluate workable alternatives to acoustic methods because of limited resolution, the optical technique analyzed still poses a significant problem because of limited range. Ranges below $3 \mathrm{~m}$ are required to obtain good results. As demonstrated by the range-error plot in Figure 4.14, ranges just beyond this have a dramatic increase in error. Through observation it is noted that laser detection is lost at approximately $4 \mathrm{~m}$. Improvements in system range are also essential for most practical applications.

\subsection{Recommendations and Improvements}

The most immediate improvement that could be made to the system studied is to develop a method for keeping some ROV lighting energized during laser operation. A narrowband filter centered at the laser wavelength might be capable of rejecting enough illumination (ideally, without unacceptably decreasing system range) that a low-light-level camera with appropriate low-power lighting could be used for vehicle piloting concurrent with laser operation. Another alternative is to employ an image subtraction method, where an image is obtained with the laser secured then subtracted from the image with the laser line. A filter technique could then be applied to the resultant image to further enhance laser detection. This would require cycling of the laser, which would reduce useful lifetime but could implemented through a mechanical window instead of power interruption.

Greater range is also essential for most practical applications. Higher laser power, receiver sensitivity or both should be implemented to increase laser detection and achieve greater ranges. Maintaining ROV position within a 1-m band is difficult especially with ocean

currents. Increasing the system's dynamic range capability along with the maximum attainable range would provide much more workable conditions for ROV deployment of this system. Alternative receivers incorporating faster auto-iris, auto-gain, or a combination could be implemented in software based on detected laser amplitude values. 
Improvements in system range-error performance could be made through better line-finding algorithms and higher image resolution. Detection of the laser light must be followed by refinement to determine only the thin line of the assumed light plane, on which system orientation is based. Using an extrema technique instead of thresholding and averaging may produce more consistent results and a corresponding increase in performance. Reducing pixel size by increasing image resolution reduces the area of object location for a given range. This provides a direct and positive improvement on range performance but requires increased processing capability.

Lastly, the system tested has no means of recording the raw video image being processed. This option would allow off-line processing of the data that could provide valuable improvements in increasing profile density. This poses a timing synchronization problem, but a video time-base corrector in conjunction with a good video recorder would provide appropriate recording quality. A slight degradation in profile accuracy would be an acceptable trade-off to achieve a much higher sampling density. 


\section{Bibliography}

[1] I. Dyer, "Fundamentals and Applications of Underwater Sound," Course Notes from 13.851, Notes from 13.851, Department of Ocean Engineering, Massachusetts Institute of Technology, 1991.

[2] C.S. Clay, H. Medwin, Acoustical Oceanography: Principles and Applications, John Wiley and Sons, New York, 1977.

[3] E.P. Zege, A.P. Ivanov, I.L. Katsev, Image Transfer Through a Scattering Medium, Springer-Verlag, New York, 1991.

[4] N.G. Jerlov, Marine Optics, Elsevier Oceanography Series, New York, 1976.

[5] J.O. Klepsvik, H.O. Torsen, K. Thoresen, "Laser Imaging Technology for Subsea Inspection: Principles and Applications," IRM/ROV 90 Conference, November 1990

[6] J.O. Klepsvik, H.O. Torsen, "Recent Advances in Accurate Underwater Mapping and Inspection Techniques," International Ocean Technology Congress, January 1989.

[7] B.K.P. Horn, Robot Vision, McGraw-Hill Book Co., New York, 1986.

[8] W.S. Ross, "Angular Spectrum Measurements of an Underwater Optical Communication Channel," Master of Science Thesis, Massachusetts Institute of Technology, 1977.

[9] S. Winther, "SPOTSCAN 2D System Description, Offshore Trials, and Test Report," Seatex A/S, Trondheim, Norway, December 1990.

[10] T. McRae, "Design and Preliminary Evaluation of an Underwater Laser-Based Imaging System," Proceedings of SPIE Ocean Optics IX, pp. 351-362, April 1988.

[11] T.H. Dixon, T.J. Pivirotto, R.F. Chapman, R.C. Tyce, "A Range Gated Laser System for Ocean Floor Imaging," MTS Journal, Vol. 17, No. 4, pp. 5-12, April 1985. 
[12] B.W. Coles, "Recent Developments in Underwater Laser Scanning Systems," Proceedings of SPIE Underwater Imaging, pp. 42-52, August 1988.

[13] H.M. Karara, "Non-Topographic Photogrammetry," Manual of Photogrammetry, pp. 785-882, American Society of Photogrammetry, Falls Church, Virginia, 1980.

[14] I. Newton, "Underwater Photogrammetry," Non-Topographic Photogrammetry, pp. 147-166, American Society for Photogrammetry and Remote Sensing, Falls Church, Virginia, 1989.

[15] J.S. Lim, Two-Dimensional Signal and Image Processing, Prentice-Hall, Englewood Cliffs, New Jersey, 1990.

[16] M.M. Thompson, "Foundations of Photogrammetry," Manual of Photogrammetry, pp. 1-36, American Society of Photogrammetry, Falls Church, Virginia, 1980.

[17] K.W. Wong, "Basic Mathematics of Photogrammetry," Manual of Photogrammetry, pp. 37-101, American Society of Photogrammetry, Falls Church, Virginia, 1980.

[18] E.J. Schimitschek, "Recent Progress in Laser Sources for Underwater Applications," Proceedings of SPIE, Ocean Optics V, pp. 38-42, August 1978.

[19] M.J. Beesley, Lasers and Their Applications, Barnes and Noble, New York, 1971.

[20] W.F. Price, J. Uren, Laser Surveying, Van Nostrand Reinhold, London, 1989.

[21] Y. Shirai, Three-Dimensional Computer Vision, Springer-Verlag, New York, 1987.

[22] Y. Shirai, M. Suwa, "Recognition of Polyhedrons with a Rangefinder," Proceedings of the 2nd International Joint Conference on Artificial Intelligence, pp. 80-87, December 1971.

[23] G. Agin, T.O. Binford, "Computer description of Curved Objects," Proceedings of the 3rd International Joint Conference on Artificial Intelligence, pp. 629-640, 1973. 
[24] G.J. Vanderburg, J.S. Albus, E. Barkmeyer, "A Vision System for Real-Time Control of Robots," Proceedings of the 9th International Symposium and Exposition on Industrial Robots, pp. 213-231, 1979.

[25] S.F. El-Hakim, "A Photogrammetric Vision System for Robots," Photogrammetric Engineering and Remote Sensing, Vol. 51, No. 5, pp. 545-552, 1985.

[26] F.B. Hildebrand, Advanced Calculus for Applications, Prentice-Hall, Englewood Cliffs, New Jersey, 1976.

[27] J. G. Baker, "Elements of Photogrammetric Optics", Manual of Photogrammetry, pp. 103-185, American Society of Photogrammetry, Falls Church, Virginia, 1980.

[28] S.F. El-Hakim, A.W. Burner, R.R. Real, "Video Technology and Real-Time Photogrammetry", Non-Topographic Photogrammetry, pp. 279-304, American Society for Photogrammetry and Remote Sensing, Falls Church, Virginia, 1989.

[29] C.G. Fox, "Description, Analysis, and Prediction of Sea-Floor Roughness Using Spectral Models," Report Number TR-279, Advanced Technology Staff, Naval Oceanographic Office, NSTL, Mississippi, 1985.

[30] W.M. Siebert, Circuits, Signals and Systems, McGraw-Hill, New York, 1986.

[31] A. V. Oppenheim, R.W. Schafer, Discrete-Time Signal Processing, Prentice-Hall, Englewood Cliffs, New Jersey, 1989. 